\title{
Composition, morphology and electrical transport properties of Co-Pb electrodeposits
}

\author{
Katalin Neuróhr ${ }^{1}$, Júlia Dégi ${ }^{1}$, Lajos Pogány ${ }^{1}$, Imre Bakonyi ${ }^{1}$, Dániel Ungvári ${ }^{2}$, \\ Kálmán $\mathrm{Vad}^{2}$, József Hakl², Ádám Révész ${ }^{3}$, László Péter ${ }^{1, *}$ \\ 1: Institute for Solid State Physics and Optics, Wigner Research Centre for Physics, \\ Hungarian Academy of Sciences. H-1525 Budapest, P.O. Box 49, Hungary \\ 2: Institute of Nuclear Research of the Hungarian Academy of Sciences. \\ H-4001 Debrecen, P. O. Box 51, Hungary \\ 3: Department of Materials Physics, Eötvös University. H-1518 Budapest, P.O.B. 32, Hungary
}

\begin{abstract}
Bath compositions were elaborated for the codeposition of $\mathrm{Co}$ and $\mathrm{Pb}$ by taking into account the chemical compatibility of $\mathrm{Co}^{2+}$ and $\mathrm{Pb}^{2+}$ with the appropriate anions. Electrolytes containing either acetate, chloride or nitrate anions were tested, but only the acetate bath proved to be appropriate for the preparation of compact $\mathrm{Co}-\mathrm{Pb}$ films. Deposits were obtained with constant current, with constant potential or by using various current and potential pulses in order to investigate the possibility of multilayer formation. The variation in deposit composition as a function of the deposition parameters was elucidated by using cyclic voltammetry, current transient measurements and gravimetry. X-ray diffraction (XRD) patterns recorded for two-pulse plated deposits revealed a nanocrystalline structure with grain sizes in the range 5 to $20 \mathrm{~nm}$. The XRD peaks could be well indexed to pure face-centered cubic $\mathrm{Co}$ and $\mathrm{Pb}$, indicating that the $\mathrm{Pb}$ codeposited with Co is not dissolved in Co but is segregated. Both the d.c.-plated and the two-pulse plated deposits exhibited anisotropic magnetoresistance without an indication for a noticeable giant magnetoresistance contribution. This means that the observed magnetoresistance arises from spindependent electron scattering events dominantly within the sufficiently large Co regions and not along electron paths between two $\mathrm{Co}$ regions via the $\mathrm{Pb}$ regions. Low-temperature resistivity measurements revealed a superconducting transition slightly below that of pure $\mathrm{Pb}$. This may be ascribed to a proximity effect: the ferromagnetic Co grains suppress somewhat the superconductivity of the $\mathrm{Pb}$ phase due to the nanoscale phase mixture of the two constituents.
\end{abstract}

Keywords: electrodeposition, $\mathrm{Co}-\mathrm{Pb}$ nanoscale mixture, magnetoresistance, superconductivity

Corresponding author. E-mail: peter.laszlo@wigner.mta.hu 


\section{Introduction}

$\mathrm{Co}$ and $\mathrm{Pb}$ are immiscible metals with very dissimilar nearest neighbor distances in the stable crystalline forms [1]. Pb has a face-centered cubic (fcc) structure at normal pressure and a hexagonal close-packed (hcp) structure at high pressure. The typical nearest neighbor distance for $\mathrm{Pb}$ is $0.35 \mathrm{~nm}$ in both forms. Co has an hep structure at room temperature that transforms to an fcc structure at $422{ }^{\circ} \mathrm{C}$. The typical nearest neighbor distance in both forms is $0.25 \mathrm{~nm}$. Based on both the immiscibility and the large difference in the nearest neighbor distances of $\mathrm{Co}$ and $\mathrm{Pb}$, it is expected that the production of either metastable $\mathrm{Co}-\mathrm{Pb}$ alloys or $\mathrm{Co} / \mathrm{Pb}$ multilayers requires highly non-equilibrium preparation techniques. Due to the lack of such studies, it is not known whether Co and $\mathrm{Pb}$ can form metastable alloys or whether a quasi-epitaxial growth is possible where one of the components stabilizes the metastable form of the other via the interaction of the interfaces.

A few reports are available only on $\mathrm{Co}-\mathrm{Pb}$ alloys and composite materials that were produced for the development of corrosion resistant Pb-rich anode materials suitable for electrowinning processes. Rashkov et al. [2] mentioned the electrodeposition technique as a tool for obtaining $\mathrm{Pb}$-rich deposits containing up to $6 \mathrm{wt} \% \mathrm{Co}$ but neither the details of the electrodeposition process nor the structural properties of the samples were given to conclude if alloy formation occurred. Nevertheless, the $\mathrm{Co}$-doped $\mathrm{Pb}$ anodes are in the focus of research in the electrowinning industry, as witnessed by a recent review of the topic [3] which also emphasizes the potential importance of electrodeposition as a production method of $\mathrm{Co}-\mathrm{Pb}$ deposits.

Yao et al. recently reported [4] on the electrodeposition of $\mathrm{Co}-\mathrm{Pb}$ dendrites from a chloride solution by d.c. plating under potentiostatic control. Although $\mathrm{PbCl}_{2}$ precipitate tends to form in cold dilute chloride solutions, the authors did not report any reactant solubility problem. No continuous $\mathrm{Co}-\mathrm{Pb}$ film was obtained for the deposition conditions used. The authors claim to have found indications by X-ray diffraction (XRD) for the formation of a metastable $\mathrm{Co}-\mathrm{Pb}$ solid solution and a segregation of the constituents upon annealing, which was accompanied by the change of the magnetic properties, too. However, in their presented XRD patterns one can see that the separation between the $\mathrm{Cu}$ substrate peak and the fcc $\mathrm{Pb}(111)$ peak is identical for the as-deposited and annealed states. This means that the line positions in the reported XRD patterns do not support the presence of a solid solution. The appearance of the hcp Co(101) line after the highest annealing temperature may rather be attributed to the growth of the Co grains that may be too small to be revealed by XRD in the as-deposited state.

Acetate-based baths were used for producing $\mathrm{Co}-\mathrm{Pb}$ nanowires in anodic alumina templates $[5,6]$. In both latter works, deposition was carried out at room temperature with an AC voltage of 40 $\mathrm{V} / 50 \mathrm{~Hz}$, but no electrochemical characterization of the deposition process was described. Although 
both transmission electron microscopy (TEM) and XRD studies have been carried out on the Co-Pb nanowires with overall compositions of $\mathrm{Co}_{82} \mathrm{~Pb}_{18}$ and $\mathrm{Co}_{48} \mathrm{~Pb}_{52}$, no quantitative data were presented to support or deny the formation of a $\mathrm{Co}-\mathrm{Pb}$ solid solution.

In the present study, the authors aimed at developing baths that are suitable for the codeposition of $\mathrm{Pb}$ and $\mathrm{Co}$ as continuous films, both in alloy and multilayer forms. Besides the chemical composition of the deposits, physical properties such as surface morphology, crystalline structure, magnetoresistance and low-temperature resistivity were also studied.

\section{Experimental}

Preliminary experiments for the codeposition of $\mathrm{Pb}$ and $\mathrm{Co}$ were performed with three different baths. Two baths were based on good solubility of both $\mathrm{Co}^{2+}$ and $\mathrm{Pb}^{2+}$ ions in the presence of acetate and nitrate anions, while the chloride bath took the advantage of the $\left[\mathrm{PbCl}_{3}\right]^{-}$complex formation at high $\mathrm{Cl}^{-}$concentration. For the acetate bath, acetic acid was also added in order to suppress the hydrolysis of the acetate ions. The bath compositions are summarized in Table 1. Since only the acetate bath could yield compact deposit that could be reliably contacted for electrical transport measurements, the reasons of the failure for the rest of the baths will be mentioned just shortly.

Electrodeposition was performed at ambient conditions in a tubular cell with an upward facing horizontal cathode of $1.5 \mathrm{~cm}^{2}$ surface area [7,8]. An EF453 type potentiostat/galvanostat served as a current source. A saturated calomel electrode (SCE) was used as reference electrode. The calomel electrode was connected to the cell via an external vessel ending in a Luggin capillary and filled up with an electrolyte having a composition identical to the bath used for deposition except that it was void of $\mathrm{Pb}^{2+}$ ions. The absence of the $\mathrm{Pb}^{2+}$ ions in the neighborhood of the reference electrode prevented the formation of $\mathrm{PbCl}_{2}$ and hence protected the calomel electrode. The counter electrode was a $\mathrm{Pb}$ wire in most cases, but a $\mathrm{Pt}$ sheet was used for solutions void of $\mathrm{Pb}^{2+}$.

The substrate was either a $\mathrm{Cu}$ sheet (preliminary experiments and sample preparation for composition analysis) or a metal-coated $\mathrm{Si}$ wafer with a $\mathrm{Cr}$ adhesive layer $(5 \mathrm{~nm})$ and a conductive $\mathrm{Cu}$ seed layer $(20 \mathrm{~nm})$. The latter $\mathrm{Si} / \mathrm{Cr} / \mathrm{Cu}$ substrate was used for all experiments where the structural or the transport properties of the deposits were studied. The electrochemical quartz crystal microbalance experiments were performed with an SRS-200 instrument and with a gold-coated quartz crystal. 
The sample composition was determined by the electron probe microanalysis (EPMA) facility of a JEOL JSM 840 scanning electron microscope. The RÖNTEC EPMA unit was operated at $25 \mathrm{kV}$. The chemical analysis was carried out for spots of about $1 \mathrm{~mm}^{2}$ surface area each. Composition data given represent averages of measurements obtained on at least three different spots for every sample.

X-ray diffraction (XRD) patterns with $\mathrm{Cu}-\mathrm{K} \alpha$ radiation were recorded on a Philips X'pert powder diffractometer in $\theta-2 \theta$ geometry. The step width was 0.02 degree in the range of 20-100 degree. No difference was seen in the diffractograms as a function of the age of the samples.

The magnetoresistance (MR) properties of the deposits were tested at room temperature in a home-built workstation. The magnetoresistance was measured with a 4-point-in-line configuration up to $H=8 \mathrm{kOe}$ external magnetic field in the field-in-plane, current-in-plane mode. The magnetoresistance was measured both in longitudinal (LMR) and transverse (TMR) configurations (when the magnetic field and the current are parallel with or perpendicular to each other, respectively). The MR ratio was defined as $\Delta R / R_{\mathrm{O}}=\left[R(H)-R_{\mathrm{o}}\right] / R_{\mathrm{O}}$ where $R(H)$ and $R_{\mathrm{O}}$ are the resistance values measured in a magnetic field $H$ and in zero magnetic field, respectively.

Low-temperature resistivity measurements were performed in the temperature range of $3.5-$ $300 \mathrm{~K}$. The temperature was controlled in a liquid-helium cryostat of type R160N by helium gas flow. The first measurements were performed at most a few weeks after the sample preparation, and the procedure was repeated four months later to test the aging of the samples. During the aging period, the samples were stored at room temperature under normal atmospheric conditions. Before both runs, the instrument was calibrated with a 50 - $\mu \mathrm{m}$-thick $\mathrm{Nb}$ sheet.

\section{Results and discussion}

\subsection{Electrochemical characterization of the baths}

Figure 1 shows cyclic voltammograms recorded for the acetate bath. During the potential scan towards the cathodic direction, the deposition of $\mathrm{Pb}$ is the first process observed since $\mathrm{Pb}$ is more noble than Co. As it can be seen in Figure 1.a, the deposition of bulk Pb starts at $-545 \mathrm{mV}$, and the diffusion-limited current density sets in at $-610 \mathrm{mV}$. In the anodic-going scan, the beginning of the dissolution of $\mathrm{Pb}$ is observed nearly at the same potential where the deposition started. Hence, the deposition of $\mathrm{Pb}$ is a reversible process.

It has been known for three decades that underpotential deposition (UPD) of $\mathrm{Pb}$ is possible on the (111) crystal face of $\mathrm{Cu}$ in the presence of various anions [9-12]. As it can be seen in Figure 
1.a, the cathodic current starts to increase prior to the bulk $\mathrm{Pb}$ deposition, and this current increase can be, at least partly, attributed to the Pb UPD process. The potential interval where the current increase occurred coincides with the observation of the UPD studies [9-12], and the same current contribution cannot be seen in the voltammograms when $\mathrm{Pb}^{2+}$ is absent. Nevertheless, the present experimental conditions are much different from the typical ones used for UPD studies, namely: (i) the $\mathrm{Cu}$ substrate is not a single crystal; (ii) the $\mathrm{pH}$ of the electrolyte is relatively high; (iii) oxygen and another metal salt (cobalt acetate) were present. Although the voltammogram in Fig. 1.a may be indicative of UPD, it has no direct consequence on the deposition after the complete coverage of the substrate with the deposit.

When the deposition of Co was studied in the acetate bath in the absence of $\mathrm{Pb}$, the deposition of Co started at $-895 \mathrm{mV}$ in the cathodic-going scan (Fig. 1.b). The anodic-going scan exhibited larger cathodic currents in the Co deposition regime, indicating that the deposition potential was strongly influenced by the nucleation overpotential of Co on the substrate. The nucleation overpotential was about the same on either $\mathrm{Cu}$ or Pt substrate. The Co deposition stopped at $-685 \mathrm{mV}$ in the anodic going scan. The Co deposition was a quasireversible process since the Co dissolution started at $-440 \mathrm{mV}$ only.

The current-potential function during the dissolution of both $\mathrm{Co}$ and $\mathrm{Pb}$ has a shape characteristic of a stripping peak in the sense that the current falls to zero very abruptly as the entire deposit is dissolved. The charge balance showed that the cathodic and anodic charges passed were equal; therefore, the deposition efficiency was nearly $100 \%$ and the metals deposited could be entirely dissolved.

When the potential scan was run in a wide potential window in the presence of both $\mathrm{Pb}^{2+}$ and $\mathrm{Co}^{2+}$ cations, the onset of $\mathrm{Pb}$ deposition was observed at the same potential as in the absence of $\mathrm{Co}^{2+}$ ions. After the diffusion-limited current density plateau of the $\mathrm{Pb}$ deposition, the current density started to increase at $-810 \mathrm{mV}$, but the slope of the curve was smaller than in the voltammogram of the Co deposition in the same potential range. The Co deposition in the potential interval of -810 to $-1075 \mathrm{mV}$ was hindered by the nucleation overpotential of $\mathrm{Co}$ on $\mathrm{Pb}$. The stronger current increase from $-1075 \mathrm{mV}$ indicated the potential range where Co-rich deposits could be formed. The reverse scan exhibited larger current densities than the cathodic-going scan, which was the result of the increase of the deposit surface (see the difference in currents for the forward and backward scans at $-0.65 \mathrm{~V}$ ). In the anodic part of the curve, the dissolution of $\mathrm{Pb}$ and Co could not be separated. The dissolution started at the same potential as in the case of the pure Pb deposit.

The interesting feature of the $\mathrm{Co}-\mathrm{Pb}$ acetate bath was that the deposition and dissolution potentials of $\mathrm{Co}$ (i.e., of the less noble element) enclose the deposition/dissolution potentials of $\mathrm{Pb}$. 
In this aspect, this bath is different than the $(\mathrm{Co}, \mathrm{Ni}, \mathrm{Fe})-(\mathrm{Cu}, \mathrm{Ag})$ baths commonly used for the electrochemical preparation of magnetic/non-magnetic multilayers where the dissolution potential of the less noble element is by far more negative than the deposition potential of the more noble element.

In contrast to the acetate bath, both the chloride and the nitrate bath were problematic. Although the deposition and dissolution potentials of both $\mathrm{Co}$ and $\mathrm{Pb}$ could be established for the chloride bath, the Co deposition took place at a very low efficiency in the high-current regime, hence making the Co deposition unreliable. From the nitrate bath, although $\mathrm{Pd}$ deposition and dissolution was possible, the reduction of the nitrate ion preceded the Co deposition. Thus, the nitrate bath proved to be unsuitable for the deposition of Co-containing alloys. Samples discussed in the further sections were all obtained with the acetate bath.

\subsection{Electrodeposition with constant polarization conditions}

It was found that compact $\mathrm{Pb}$ deposits can be obtained with the constant current mode when the current density is lower than the diffusion-limited current density of the $\mathrm{Pb}$ deposition (note that in this current density range, no Co codeposition occurred). The increase of the current density above the diffusion-limited current density of the $\mathrm{Pb}$ deposition changed the morphology of the deposit. As opposed to the compact $\mathrm{Pb}$ deposits obtained with low current density, the deposits became dendritic, still without the inclusion of $\mathrm{Co}$ as it turned out from the composition analysis. No compact deposits could be produced between about 0.9 to 10 times of the diffusion-limited $\mathrm{Pb}$ deposition current density. The aspect ratio of the $\mathrm{Pb}$ crystals in this current density regime was extremely high. The dendrite structure was so weak that after the removal of the electrolyte the dendrite system collapsed and became oxidized in air, leading to a loosely adherent white powder. The $\mathrm{Pb}$ crystals could be imaged in an optical microscope under liquid without drying. The deposits were rinsed several times with distilled water without removing the protective fluid drop from the dendrite system. As it can be seen in Figure 2, the length of the $\mathrm{Pb}$ dendrites ranged to several tens of micrometers while their diameter was mostly below $1 \mu \mathrm{m}$. The dendrites grown showed side branches with fourfold symmetry (sidewise growing crystals incline at a right angle to the main branch).

Compact deposits were obtained again with at least 10 times larger current density than the diffusion-limited current density of $\mathrm{Pb}$. In this regime, the Co content of the deposits was typically above 80 at.\%. Therefore, a wide composition interval of the deposit could not be achieved with a simple d.c. deposition at constant current. 
It was also investigated whether electrodeposition at constant potential is appropriate to obtain compact $\mathrm{Co}-\mathrm{Pb}$ deposits. Well-adhering deposits were obtained between $-0.58 \mathrm{~V}$ and $-1.10 \mathrm{~V}$ deposition potentials. The deposits obtained in this potential range did not contain any Co. The cathodic current increased as the deposition potential became more negative, but this could be fully ascribed to a change in the deposit morphology and the resulting change in the ion transport rate in the electrolyte. Figure 3.a shows that granular type deposits were obtained at the potential where the deposition became diffusion-limited $(E=-0.65 \mathrm{~V})$. At more negative potentials, the deposit was composed of $\mathrm{Pb}$ dendrites, which is demonstrated with the SEM picture of a sample produced at $E=-1.025 \mathrm{~V}$ (Fig. 3.b). As long as the Co codeposition did not start, the adherence of the sample to the surface was sufficient and the system of the $\mathrm{Pb}$ crystals was strong enough to hold the deposit on the substrate, albeit the SEM picture clearly shows the presence of voids between the elongated $\mathrm{Pb}$ crystals. At potentials where the codeposition of Co started (-1.075 V, see Fig. 1.c), the percolation of the $\mathrm{Pb}$ dendrites and their adherence to the substrate surface became so weak that no continuous sample could be obtained. Therefore, the potentiostatic growth mode exhibited a similar gap in the deposit composition as observed also with the galvanostatic conditions: by using d.c. plating, either pure $\mathrm{Pb}$ or Co-rich deposits with about 80 at.\% Co could only be obtained.

\subsection{Electrodeposition with two-pulse plating}

In order to bridge the composition gap and also to investigate whether layered $\mathrm{Co} / \mathrm{Pb}$ samples can be produced, two-pulse plating was tested with the acetate bath to obtain $\mathrm{Co}-\mathrm{Pb}$ deposits.

Two-pulse plating was performed with the galvanostatic/potentiostatic $(\mathrm{G} / \mathrm{P})$ method $[7,13]$. The deposition in the high-current $G$ pulse took place with such a large current density that would otherwise be able to produce continuous, compact Co-rich layers in the d.c. deposition mode. In the $\mathrm{P}$ pulse, $\mathrm{Pb}$ was deposited at various potentials where Co could neither deposit nor dissolve. The selection of the appropriate $\mathrm{Pb}$ deposition potential was based on the optimization method elaborated for $\mathrm{Co} / \mathrm{Cu}$ multilayers [14]. While the pulse length was regulated with the pulse time in the $\mathrm{G}$ mode, a real-time current integration was performed during the P pulse in order to switch to the next pulse after passing a predetermined amount of charge, hence keeping the amount of deposit constant for the P pulse regardless of the actual current.

Figure 4 presents all data related to the optimization of the $\mathrm{Pb}$ deposition potential $\left(E_{\mathrm{Pb}}\right)$. It can be seen in Fig. 4.a that in the potential range between $-0.55 \mathrm{~V}$ and $-0.63 \mathrm{~V}$, the deposit composition is independent of the $\mathrm{Pb}$ deposition potential. If $E_{\mathrm{Pb}}$ was more negative than $-0.63 \mathrm{~V}$, the Co content of the deposit started to increase. This was because the deposition of Co could 
continue on the Co-rich deposit surface produced in the preceding pulse. On the other hand, the $\mathrm{Pb}$ content of the deposit increased drastically when $E_{\mathrm{Pb}}$ was less negative than $-0.55 \mathrm{~V}$. The current transients recorded for the $\mathrm{Pb}$ pulse (Fig. 4.b) show that the increase of the $\mathrm{Pb}$ content of the deposit cannot be associated with a Co dissolution process. The current was negative throughout the $\mathrm{Pb}$ deposition pulse, regardless of $E_{\mathrm{Pb}}$. The lack of an anodic transient in the chronoamperometric curves recorded for the $\mathrm{P}$ pulse is in accord with the dissolution potential of Co observed in the cyclic voltammograms (see Fig. 1). The increase of $\mathrm{Pb}$ content for $E_{\mathrm{Pb}}>-0.55 \mathrm{~V}$ is due to the fact that $\mathrm{Pb}$ deposition in this potential range does not lead to the depletion of the electrolyte near the cathode surface since $\mathrm{Pb}$ deposition takes place at a much lower rate than the diffusion-limited current density. Therefore, a substantial portion of the charge passed during the upcoming $\mathrm{G}$ pulse led also to $\mathrm{Pb}^{2+}$ reduction, and Co deposition could start only after the electrolyte was depleted for the $\mathrm{Pb}^{2+}$ ion. The inset of Figure 4.b also shows that the steady-state current density recorded at the end of the $\mathrm{P}$ pulse is in very good agreement with the value obtained during the slow potentiodynamic scan. This latter fact supports the assumption that Co is practically inert in the potential range studied for sake of the optimization of the $\mathrm{Pb}$ deposition.

The change in the morphology of the deposit as a result of the modification of the $\mathrm{Pb}$ deposition potential can be seen in Figure 5. The more negative was the deposition potential during the P pulse, the more compact deposit could be obtained. SEM secondary electron images (not shown) indicated that, along with the fairly even morphology, the distribution of the deposit components was also the better, the more negative $E_{\mathrm{Pb}}$ value was selected. For $E_{\mathrm{Pb}}>-0.56 \mathrm{~V}$, dendrites appeared at the deposit surface. Therefore, further experiments were performed with $E_{\mathrm{Pb}}=$ $-0.62 \mathrm{~V}$ as an optimum value.

The current density during the $\mathrm{G}$ pulse of the two-pulse plating was also optimized. The composition of the resulting samples is shown in Figure 6. Changing the current density in the range -10 to $-50 \mathrm{mAcm}^{-2}$ (by keeping the total charge for the G pulse constant in the sample series) resulted in samples with identical composition within the experimental error. The surface morphology of the deposits was very similar to that shown in Fig. 5.a, regardless of the current density. The optimum conditions for the current density in the G pulse were selected on the basis of the mechanical and electrical behavior of the samples. Both high $\left(|j|>35 \mathrm{mAcm}^{-2}\right)$ and low $(|j|<20$ $\mathrm{mAcm}^{-2}$ ) cathodic current densities in the G pulse led to fragile specimens on which the electrical contacts during the transport measurements were uncertain. Therefore, $j=-32 \mathrm{mAcm}^{-2}$ was chosen as an optimum value.

It was also checked if a change in the pulse parameters has an impact on the deposit composition. In this study, both the composition of the deposits and the weight change on the 
substrate were measured. Both the growth rate and the composition of the deposits were calculated by assuming constant values for the current efficiency during the P pulse $\left(\eta_{\mathrm{P}}\right)$, the current efficiency in the $\mathrm{G}$ pulse $\left(\eta_{\mathrm{G}}\right)$ and the molar fraction of $\mathrm{Pb}$ in the deposit produced with the $\mathrm{G}$ pulse $\left(y^{*} \mathrm{~Pb}\right)$. Figure 7 shows the experimental data and the result of calculations. The best agreement between the experimental data and the calculations was obtained with the following parameters: $\eta_{\mathrm{P}}=1, \eta_{\mathrm{G}}=$ 0.97 and $y^{*}{ }_{\mathrm{Pb}}=0.1$.

The properties of the two-pulse plated samples were also tested with an anodic linear sweep voltammetry experiment in parallel with the measurement of the deposit weight by using a quartz crystal microbalance. After finishing the deposition of the sample, a slow anodic-going potential scan was started. A representative pair of curves is presented in Figure 8. The dissolution of the pulse-plated $\mathrm{Co}-\mathrm{Pb}$ sample takes place in two stages. The first stripping peak starting at $-510 \mathrm{mV}$ corresponds to the dissolution of lead. After the decay of the current, no other process is seen until the potential reaches $-380 \mathrm{mV}$. From $-380 \mathrm{mV}$ on, the dissolution of Co (or the Co-rich part of the sample) takes place. The dissolution of Co from the pulse-plated sample takes place at a more positive potential than that observed in the cyclic voltammograms, where the start of the dissolution of pure Co was observed from $-440 \mathrm{mV}$ (see Fig. 1.b).

Alloying of $\mathrm{Co}$ with $\mathrm{Pb}$ is expected to shift the Co dissolution potential to the negative direction, since the dissolution potential of $\mathrm{Pb}$ is more negative than that of Co. Therefore, alloying cannot explain the change of the dissolution potential. It can rather be assumed that a curvature effect is responsible for the shift of the dissolution potential to the positive direction. If the dissolution of $\mathrm{Pb}$ leaves behind a Co sponge with concave surface, the dissolution is expected to occur at a more positive potential than for a flat Co surface. A similar effect was found for metastable Co-Ag alloys [15]. The curvature effect on the dissolution potential has also been described recently with theoretical considerations for nanoparticles [16], obviously with an opposite change of dissolution potential due to their convex surface. The change in dissolution potential of Co implies that the curvature radius is near to at most a few tens of nanometers. The fact that the dissolution potential of $\mathrm{Pb}$ is the same as that of pure $\mathrm{Pb}$ deposits indicates that no alloy formation took place; otherwise the $\mathrm{Pb}$ dissolution should be described with the term of quasi-critical potential of dissolution [17].

The comparison of the charge passed during the observation of the more negative stripping peak with the corresponding weight change observed with the QCM yields the result that it should account for the dissolution of pure $\mathrm{Pb}$. However, the weight change observed for the less negative stripping peak is too large to be caused by merely a pure Co phase. The charge and weight balance is only satisfied if it is assumed that the second stripping peak accounts for the dissolution of a Co- 
rich deposit with some $\mathrm{Pb}$ content and $y^{*} \mathrm{~Pb}=0.075$. This value is in an acceptable agreement with that found in the composition analysis (see two paragraphs above). Nevertheless, it should be noted that the results of EQCM measurements cannot give any hint at whether the $\mathrm{Pb}$ atoms codeposited with Co during the G pulse are dissolved atomically in Co (such as to form an alloy) or constitute a pure $\mathrm{Pb}$ phase (either in the form of small inclusions in the Co matrix or separate $\mathrm{Pb}$ grains between the larger $\mathrm{Co}$ grains). The dissolution of both $\mathrm{Pb}$ and $\mathrm{Co}$ at two distinct potential intervals and the fairly good agreement of the charge to weight change ratio with the literature data of the constituent elements indicate that both the $\mathrm{Pb}$ and $\mathrm{Co}$ phase form a percolating network without any significant inclination to layer formation. This preliminary conclusion will be strongly underpinned by both the XRD and MR data (see the forthcoming sections).

\subsection{XRD study of two-pulse plated samples}

A series of 14 samples deposited from the acetate bath by the G/P pulse combination were investigated with XRD. In analyzing the XRD data, we should keep in mind that, in terms of the above discussions, a Co-rich $\mathrm{Co}-\mathrm{Pb}$ deposit is formed during the $\mathrm{G}$ pulse. More specifically, according to Section 3.3, the amount of $\mathrm{Pb}$ codeposited with $\mathrm{Co}$ in this pulse is about 10 at.\%. An important aim of the present XRD study was to obtain information on whether the Co-rich deposit is a $\mathrm{Co}-\mathrm{Pb}$ alloy (metastable solid solution) or just a mixture of $\mathrm{Co}$ and $\mathrm{Pb}$ grains. On the other hand, the deposition conditions chosen ensure that pure $\mathrm{Pb}$ is deposited during the $\mathrm{P}$ pulse in the two-pulse plated samples.

For the samples prepared for the XRD studies, the deposition potential during the $\mathrm{P}$ pulse was varied between $-0.595 \mathrm{~V}$ and $-0.656 \mathrm{~V}$ and it was in most cases the optimized value $(-0.62 \mathrm{~V})$. The current density during the $G$ pulse was varied between -24 and $-48 \mathrm{mAcm}^{-2}$ and it was in most cases $-32 \mathrm{mAcm}^{-2}$. The equivalent layer thicknesses of deposits formed during both pulses were varied between 1 and $6 \mathrm{~nm}$ by properly controlling the charge passing through the cell (P pulse) or the current density or pulse duration ( $\mathrm{G}$ pulse). For comparison, a d.c. plated pure $\mathrm{Pb}$ deposit obtained under potentiostatic control and a d.c. plated Co-rich deposit obtained under galvanostatic control and having an overall $\mathrm{Pb}$ content of about 20 at.\% was also prepared under the conditions previously optimized for two-pulse plating.

A typical diffractogram for the two-pulse plated samples can be seen in Fig. 9. The common features of the diffractograms of the two-pulse plated deposits can be summarized as follows.

The diffractograms are dominated by the diffraction lines that belong to fcc $\mathrm{Pb}$. Several diffraction lines characteristic of the stable fcc crystal structure of $\mathrm{Pb}$ can be easily identified in the 
diffractogram. Although a weak (111) texture could be identified (the intensity ratio $I_{111} / I_{100}$ was about 10, as opposed to 2.5 observed in powder diffractograms), the abundance of fcc $\mathrm{Pb}$ lines in the diffractogram indicates that $\mathrm{Pb}$ crystallites with various orientations are present in the deposits. There were some other peaks also visible in the XRD patterns (Fig. 9) which can be assigned to the known diffraction peaks of fcc and/or hep Co. Unfortunately, the assignable fcc and hcp Co peaks strongly overlap either with some of the diffraction lines of fcc $\mathrm{Pb}$ or with each other. Therefore, it is not straightforward to make an unambiguous assignment as to whether the observable Co lines correspond to the fcc or hcp form of Co. A further help comes from the fact that the hcp $\operatorname{Co}(100)$ reflection the position of which $\left(2 \Theta=41.589^{\circ}\right)$ is not overlapping with any $\mathrm{Pb}$ or Co lines is practically missing from the XRD patterns or it is at least definitely much weaker than the observed other Co lines as it can be seen in Fig. 9 (note the logarithmic intensity scale). Therefore, it is reasonable to assign the observed Co lines to the fcc phase.

Qualitatively, the diffractogram of the d.c. plated $\mathrm{Pb}$ deposit was very similar as far as the appearance of the fcc $\mathrm{Pb}$ lines is concerned (evidently, the Co lines were missing here). For the d.c. plated Co-rich deposit, the XRD pattern contained both the $\mathrm{Pb}$ and Co lines, just as in the two-pulse plated samples; however, in the d.c. plated sample, due to the much smaller overall $\mathrm{Pb}$ content of the deposit, the $\mathrm{Pb}$ lines were less intense with respect to the Co lines.

For a quantitative evaluation of the XRD patterns, each clearly visible peak was fitted with a Pearson-VII function from which the peak position and the peak width were deduced. For each diffraction line, the lattice constant was determined from the peak position. Since several $\mathrm{Pb}$ reflections were visible for each sample, it was reasonable to carry out an extrapolation to $\Theta=90^{\circ}$ in order to get the fcc lattice constant $a$ more accurately by plotting $a$ against $\cos \Theta \cdot \operatorname{ctg} \Theta[18]$.

By using this procedure, the fcc lattice parameter of the d.c. plated pure $\mathrm{Pb}$ deposit was $0.49498 \mathrm{~nm}$ which value differs by $0.01 \%$ only from the bulk value of $\mathrm{Pb}(0.49502 \mathrm{~nm}[1])$. The fcc $\mathrm{Pb}$ lattice parameter values deduced for the two-pulse plated samples fell in the range 0.49494 $\mathrm{nm}$ to $0.49598 \mathrm{~nm}$ but mostly being only slightly larger (by typically 0.00010 to $0.00020 \mathrm{~nm}$ ) than the bulk $\mathrm{Pb}$ value and not showing any systematic variation with equivalent layer thicknesses deposited during either of the pulses. We can thus conclude that the measured lattice parameters demonstrate the formation of a pure fcc $\mathrm{Pb}$ phase not only during the $\mathrm{P}$ pulse but also during the $\mathrm{G}$ pulse when $\mathrm{Co}$ and $\mathrm{Pb}$ are codeposited. Since the atomic size of $\mathrm{Co}$ is much smaller than that of $\mathrm{Pb}$, a $\mathrm{Pb}$ phase with substitutional $\mathrm{Co}$ atoms should exhibit a reduced lattice parameter with respect to bulk $\mathrm{Pb}$. By contrast, rather lattice parameter values slightly larger than that of bulk $\mathrm{Pb}$ were measured (probably due to experimental uncertainty) for the fcc Pb phase. This means that the fcc $\mathrm{Pb}$ phase occurs in very pure form in the deposits, in agreement with the known negligible 
equilibrium solubility of $\mathrm{Co}$ in $\mathrm{Pb}[1]$ and with the deposition conditions. Of course, since XRD provides overall information only on the sample, the presence of a small amount (at most a few percent of the total $\mathrm{Pb}$ volume) of a $\mathrm{Pb}$ phase with some dissolved $\mathrm{Co}$ cannot be excluded from these XRD measurements.

As noted above, the Co phase present in the samples can be tentatively assigned to the fcc crystal structure. Therefore, the XRD lines belonging to Co were evaluated along this line. Apart from the samples with equivalent Co layer thickness below $2 \mathrm{~nm}$, for most of the samples also the fcc $\mathrm{Co}(220)$ and/or the fcc $\mathrm{Co}(311)$ lines could be evaluated in addition to the main fcc $\mathrm{Co}(111)$ line. In such cases, the same extrapolation procedure was used for deriving the lattice parameter of fcc $\mathrm{Co}$ as applied for $\mathrm{fcc} \mathrm{Pb}$ above whereas for the rest of the samples, the lattice parameter was evaluated from the main fcc $\operatorname{Co}(111)$ line only. As a result, lattice parameter values very close to the bulk fcc Co value $(0.35446 \mathrm{~nm}$ [1]) were obtained for all two-pulse plated samples. The majority of the measured values falls within about $0.00030 \mathrm{~nm}$ of the bulk fcc Co value whereas most of the values with much larger deviation belong to samples where a single Co peak could only be used and, thus, the lattice parameter determination was less accurate in these latter cases.

By taking the bulk fcc $\mathrm{Pb}$ and Co lattice parameters, the Vegard's law allows us to estimate that a deviation of $0.00015 \mathrm{~nm}$ from the bulk value corresponds to maximum 0.1 at.\% substitutional alloying for either of the main components. The lattice parameter data lead in this manner to the conclusion that the fcc $\mathrm{Pb}$ phase does not contain any $\mathrm{Co}$ whereas the fcc Co phase may contain at most 0.2 at.\% of dissolved $\mathrm{Pb}$. This means that all the samples consist of a phase mixture of pure $\mathrm{Pb}$ and almost pure Co, meaning a strong segregation of the two elements in agreement with their known mutual immiscibility [1]. Of course, there may be a very small volume fraction (a few percent) of a Co-rich phase with higher amount of dissolved $\mathrm{Pb}$ present since this would be below the detection limit of XRD. The observed segregation is also in strong agreement with the facts that the dissolution of the phases produced takes place in distinct potential intervals that can be related, with some correction to the curvature effect as shown in Sec. 3.3, to the dissolution of the pure components.

Usually, deposits obtained by two-pulse plating from a bath containing ions of a more noble and a less noble metal exhibit a multilayered structure [13] such as, e.g., in the Co-Cu system. The situation is quite different in the Co- $\mathrm{Pb}$ system even if we apply the same $\mathrm{G} / \mathrm{P}$ pulse combination where the deposition potential of the more noble component $(\mathrm{Pb})$ is optimized as described in Section 3.3. The reason is that here, whereas a pure $\mathrm{Pb}$ deposit is formed during the $\mathrm{P}$ pulse, a complete segregation of $\mathrm{Pb}$ and $\mathrm{Co}$ occurs during the $\mathrm{G}$ pulse applied for producing a magnetic layer. This segregation is clearly shown by the XRD data. The fraction of Co grains and $\mathrm{Pb}$ grains 
(in terms of atomic fractions) formed during each $\mathrm{G}$ pulse is about 0.9 and 0.1 , respectively. Due to the hindered nucleation of $\mathrm{Co}$ on $\mathrm{Pb}$, the deposit formed during the $\mathrm{G}$ pulse certainly consists of larger $\mathrm{Co}$ grains whereas the growth of $\mathrm{Pb}$ continues on the previous $\mathrm{Pb}$ surface on areas where $\mathrm{Co}$ cannot nucleate. This definitely leads to an uneven growth of the deposit. This uneven growth is further amplified in the next pulse due to the hindered nucleation of $\mathrm{Pb}$ on the Co islands. Finally, we end up with a structure in which a percolating pure $\mathrm{Pb}$ network is separated by Co or Co-rich grains. Evidently, such a deposit is not expected to exhibit diffraction features characteristic of a multilayer structure since a structural coherence in the growth direction does not develop. This is in complete agreement with the occurrence of various crystal orientations as well as the absence of satellite reflections typical for multilayers [19].

From the line width of the individual XRD peaks, the grain size can also be estimated by using Scherrer's formula [18] and this was done by using the broadening of the strongest fcc $\mathrm{Pb}(111)$ and fcc $\mathrm{Co}(111)$ lines. For the fcc Pb phase, the higher-index reflections (200), (220), (311) and (222) were still of sufficient intensity and these lines exhibited somewhat larger line widths than the main (111) line, indicating the presence of microstrains. Therefore, the use of Scherer's formula underestimates the grain size to some extent and, thus, the actual grain sizes can be somewhat larger than those to be presented here. For the fcc Co phase, the situation is similar though here the main (111) line has only a sufficient intensity for a reliable determination of the broadening.

The grain sizes determined according to the above procedure for the fcc $\mathrm{Pb}$ phase were about $19 \mathrm{~nm}$ (with a scatter of $1 \mathrm{~nm}$ ) for the two-pulse plated samples and the d.c. plated pure $\mathrm{Pb}$ deposit. The only exception was the two-pulse plated sample with an equivalent $\mathrm{Pb}$ layer thickness of $1 \mathrm{~nm}$. For the fcc Co phase, the grain sizes obtained were about 6 to $7 \mathrm{~nm}$ for the two-pulse plated deposits with an equivalent Co layer thickness of $3 \mathrm{~nm}$ whereas the grain size decreased drastically when the equivalent Co layer thickness was reduced down to below $1 \mathrm{~nm}$.

The grain size deduced from the line broadening data refers to the thickness of the coherently scattering regions. By taking into account the XRD measurement geometry, this implies that the grain size deduced refers to crystallite dimensions along the growth direction (i.e., perpendicular to the substrate) and does not give any information for the lateral dimension of the grains. In view of this, we can understand that the "grain size" of the Co phase (i.e., the "thickness" of the Co regions) is much less than that of the $\mathrm{Pb}$ phase. Indeed, the effective thickness of the latter can be much larger than the equivalent thickness deposited during the $\mathrm{P}$ pulse since a $\mathrm{Pb}$ phase is formed between the Co regions also during the $\mathrm{G}$ pulse. This provides a percolation of the $\mathrm{Pb}$ regions through the layers deposited during the $\mathrm{G}$ pulse. Whichever equivalent layer thickness $(\mathrm{Pb}$ 
or $\mathrm{Co}$ ) was chosen to be near to or below $1 \mathrm{~nm}$, a grain size reduction was observed for the corresponding crystals.

It was demonstrated by Michaelsen [19] that in a nanoscale mixture of $\mathrm{Co}$ and $\mathrm{Cu}$ grains, a single fcc lattice parameter is taken by both phases, which implies a coherent matching of the two kinds of grains at the interfaces. Apparently, a prerequisite for this matching is not only the common crystal structure of the two phases but also a relatively small difference in the lattice constant values and a significantly large stiffness of both lattices. For the $\mathrm{Co}$ and $\mathrm{Cu}$ metals, the lattice mismatch is about $2 \%$ only whereas this quantity between $\mathrm{Pb}$ and $\mathrm{Co}$ amounts to about 30 $\%$. Evidently, this large lattice mismatch cannot lead to the coherent formation of a network of $\mathrm{Pb}$ and Co grains and, therefore, the bulk lattice constants (lattice-plane distances) of both constituent phases determine the observed XRD pattern. Obviously, due to the soft lattice of $\mathrm{Pb}$, the large lattice mismatch is relaxed towards the $\mathrm{Pb}$ grain interior on a fairly small length scale. This also implies that the interfaces between the grains of the two different phases will be highly nonequilibrium. As it turned out in previous sections, this often leads to such a low level of matching between the grains of the two metals that the whole deposit can easily disintegrate and compact deposits could only be obtained under very specific conditions.

\subsection{Magnetotransport measurements}

Typical magnetoresistance curves for d.c. plated and two-pulse plated samples prepared with the acetate bath are shown in Figure 10.

For all samples investigated, the room-temperature magnetoresistance measurements revealed anisotropic magnetoresistance (AMR). The anisotropic magnetoresistance is characterized by positive LMR and negative TMR. The magnitude of the AMR is defined [20] as the difference LMR-TMR in the saturation region of the $M R(H)$ curves. The observed clear AMR behavior, a characteristic of ferromagnetic metals and alloys, indicates the presence of a ferromagnetic (FM) phase in the sample. An AMR behavior can be observed if the size of the FM regions present is larger than the electron mean free path in the bulk form of the FM phase. The FM regions can be either completely isolated in a non-magnetic matrix if they are sufficiently large or they can form a percolating network of smaller FM regions.

The $M R(H)$ curves in Fig. 10.a reveals that the AMR value of pure Co ("d.c. plated Co") deposited from the acetate bath void of $\mathrm{Pb}^{2+}$ is about $0.75 \%$. On the other hand, a roomtemperature AMR of about $2 \%$ was reported for Co films electrodeposited from a sulfate bath [21] and for evaporated hcp Co films [22]. Since the "d.c. plated Co" sample in the present study was 
pure Co, the reduced AMR value in comparison with standard values of bulk Co should be ascribed to the fine-grained structure of this deposit. For small grain sizes, the volume fraction of grain boundaries increases and the increased contribution of grain boundary scattering results in a larger background resistivity $R_{0}$. Thus, when calculating the ratio $\Delta R / R_{0}$, the magnetoresistance is reduced.

According to the $M R(H)$ curves measured for "d.c. plated Co-Pb" (Fig. 10.b), when Pb was codeposited with Co, the AMR is further reduced down to about $0.35 \%$ for all d.c. plated samples in the current density range applied. Since no alloy formation could be established from the XRD data, the codeposited $\mathrm{Pb}$ appears as a second phase ( $\mathrm{fcc} \mathrm{Pb}$ ) in the deposit in which Co is a majority phase (the overall composition of the deposit is about 80 at.\% Co and 20 at.\% $\mathrm{Pb}$ ). By noting that the room-temperature resistivity of fcc $\mathrm{Pb}$ is $21.0 \mu \Omega \mathrm{cm}$ [23] and that of hcp Co is $5.8 \mu \Omega \mathrm{cm}$ [23] whereas for fcc Co this value is $5.5 \mu \Omega \mathrm{cm}$ [24], the appearance of a second phase with a much larger resistivity definitely increases the overall resistivity of the $\mathrm{Co}-\mathrm{Pb}$ deposit with respect to a pure Co deposit under otherwise identical deposition conditions. The two-phase nature of the deposit itself further increases the background resistivity on the one hand and, due to the presence of a non-FM phase in the deposit, the volume fraction of the FM phase is reduced on the other hand. All these facts arising due to the codeposition of a $\mathrm{Pb}$ phase to the Co matrix are expected to result in a diminution of the AMR magnitude. As discussed in the previous section, the large lattice mismatch at the interfaces between the $\mathrm{Pb}$ and $\mathrm{Co}$ grains results in a highly non-equilibrium grainboundary structure, also contributing to a larger background resistivity.

The $M R(H)$ curves measured for "two-pulse plated Co-Pb" (Fig. 10.c) also reveals AMR behavior. The AMR of the two-pulse plated sample was $0.45-0.50 \%$, i.e., slightly higher than that of the AMR of the "d.c. plated Co-Pb" sample. Nevertheless, the clear AMR character of the $M R(H)$ curves for the two-pulse plated $\mathrm{Co}-\mathrm{Pb}$ deposits evidences that the magnetic phase (essentially $\mathrm{Co}$ ) deposited in the $\mathrm{G}$ pulse consists of either large individual Co regions or a percolating network of smaller Co regions. The AMR character of the magnetoresistance curves is in agreement with the finding of the structural study that no multilayer formation took place in the two-pulse plated samples.

Both the d.c. plated and the two-pulse plated deposits exhibited anisotropic magnetoresistance without an indication for a noticeable giant magnetoresistance contribution. This means the observed magnetoresistance arises from spin-dependent electron scattering events dominantly within the sufficiently large Co regions and not along electron paths between two Co regions via the $\mathrm{Pb}$ regions

As to the differences in the AMR magnitude of the above discussed three samples, we have referred to possible differences in the room-temperature zero-field resistivity of these samples. 
Unfortunately, the rather porous nature of the $\mathrm{Co}-\mathrm{Pb}$ samples prevented us, in spite of some efforts on this, from measuring a reliable value for their zero-field resistivity, especially concerning any systematic change with deposition conditions or equivalent layer thicknesses. It was observed nevertheless that, in general, two-pulse plating resulted in more compact deposits than d.c. plating.

\subsection{Low-temperature resistivity measurements on two-pulse plated samples}

Four samples prepared with two-pulse plating by varying the length of the high-current pulse (i.e., the effective Co layer thickness) were investigated by low-temperature electrical transport measurements down to the liquid-He range with special interest for observing a superconducting transition of the $\mathrm{Pb}$ phase. The preparation parameters are summarized in Table 2. These samples are practically equivalent to those which were prepared for XRD studies with varying Co layer thickness.

The room-temperature resistivity of these samples was also attempted to be determined and the measured values ranged between 5 and $16 \mu \Omega \mathrm{cm}$, again without any systematic change, probably due to the low accuracy of the measurements, with the equivalent Co layer thickness (Table 2). With reference to the data quoted in the previous section, these values fall between the bulk $\mathrm{Co}$ and $\mathrm{Pb}$ values. However, these measured values may be eventually too small by considering that $\mathrm{XRD}$ indicated a nanoscale mixture of $\mathrm{Co}$ and $\mathrm{Pb}$ regions and in such a case a fairly large resistivity can also be expected as discussed in the previous section. In any case, the rather porous nature and high surface roughness of the deposits does not allow achieving reliable resistivity data on these samples. Due to the same problems, contacting the samples for the lowtemperature measurements was also not a straightforward task and the contacts were often broken during the cooling or heating runs. Since the deposits were quite soft and hence the electrical contacts caused some deformation, normalized data could be reliably compared only.

The results of low-temperature resistivity measurements indicated that the temperature evolution of the resistivity was very similar for all samples down to the onset of a superconducting transition $\left(T_{\mathrm{c}}\right)$. This is demonstrated in Fig. 11 which shows the measured data for the four samples on a log-log scale whereas the insert displays the resistivity for sample C on a lin-lin scale. In both cases, the experimental results are presented in the form of normalized resistance data $R_{\text {norm }}=[R(T)$ $-R(0)] / R(300 \mathrm{~K})$ where $R(T)$ is the sample resistance at temperature $T, R(0)$ is the residual resistance extrapolated from the data at the lowest temperatures in the normal state.

A common behavior of resistivity with temperature, including the onset of a superconducting state, is expected since all samples consisted of a mixture of metallic nanoscale Co 
and $\mathrm{Pb}$ regions. Above $T_{\mathrm{c}}$, the resistivity exhibits the typical temperature dependence characteristic of metals. As the insert of Fig. 11 indicates, the resistivity is already practically constant at the lowest temperatures before reaching the superconducting state.

This can be more clearly seen in Fig. 12 for sample B which shows the experimental data in the vicinity of the superconducting transition only. There is definitely a downward shift in $T_{\mathrm{c}}$ with respect to the pure bulk $\mathrm{Pb}$ value $\left(T_{\mathrm{c}}=7.2 \mathrm{~K}[23]\right)$ by about $0.2 \mathrm{~K}$. One can also observe some aging effect in that the transition to the superconducting state proceeded differently for the freshly prepared sample (i.e., with the measurement carried out a few weeks after the sample preparation) and for repeating the measurement after some four months of aging at room temperature. We can also see that two transition temperatures can be identified for both the fresh and the aged samples. One can also observe that, at least for the fresh state, not only the $\mathrm{Pb}$ phase but the whole sample is in a superconducting state below about $5.8 \mathrm{~K}$ whereas the aged state does not reach yet the fully superconducting state at least down to $4.4 \mathrm{~K}$.

Similar superconducting transitions with $T_{\mathrm{c}}$ values below that of bulk $\mathrm{Pb}$ were observed in each sample and a two-step transition as well as an aging effect was also observed in each case. For samples B, C and D, the deposits "relaxed out" of the superconducting state in that the transition temperature of the second stage decreased strongly (see Fig. 11 for sample B). Interestingly, sample A showed a different behavior concerning superconductivity (Fig. 13). Namely, no superconducting transition could be observed for this sample in the fresh state but rather a resistance minimum at about $6.3 \mathrm{~K}$ (resembling a Kondo effect) whereas the aged state exhibited a superconducting transition with $T_{\mathrm{c}}=6.5 \mathrm{~K}$, i.e., by $0.7 \mathrm{~K}$ below that of bulk $\mathrm{Pb}$. The strange behavior of sample A is not clear. From the deposition conditions we can infer that the only difference between sample A and sample B is in the equivalent layer thickness of the Co-rich deposit prepared during the G pulse (3.0 $\mathrm{nm}$ and $2.15 \mathrm{~nm}$, respectively).

The origin of the reduced superconducting transition temperature in the $\mathrm{Co}-\mathrm{Pb}$ deposits can be ascribed to a magnetic proximity effect. It has been shown namely for ferromagnetic/superconducting layered structures (e.g., Fe/V [25,26]) that an intimate contact between the ferromagnetic and superconducting layers reduces the superconducting transition temperature of the superconducting phase through the pair-breaking effect of the spin-polarized electrons of the ferromagnet penetrating into the superconductor. Since data in the previous sections indicated that our $\mathrm{Co}-\mathrm{Pb}$ deposits consist of a nanoscale mixture of $\mathrm{Co}$ and $\mathrm{Pb}$ regions, this magnetic proximity effect should be effective also in this case by considering that the superconducting coherence length is about $80 \mathrm{~nm}$ in bulk $\mathrm{Pb}$ [23] whereas the $\mathrm{Pb}$ grain size was 
found to be of the order of a few tens of a nanometer. The degree of $T_{\mathrm{c}}$ suppression may vary with the actual relative topology of the ferromagnetic and superconducting phase. Along this line, the aging effects observed indicate that there may be a slight room-temperature structural relaxation of the relative arrangement of the two components of the constituent phases, especially in the grain boundaries which provide the intimate contact for the ferromagnet and the superconductor. There are theoretical calculations indicating the influence of the junction interface intermixing on the $T_{\mathrm{c}}$ suppression [27].

\section{Conclusions}

Several bath formulations have been tested for the codeposition of $\mathrm{Co}$ and $\mathrm{Pb}$. It was revealed that no compact deposits can be obtained with a Co molar fraction below 0.8 in either current-controlled or potential-controlled mode because $\mathrm{Pb}$ is prone to develop dendrites. Compact deposits have been obtained from the acetate bath by pulsed electrodeposition using a G/P pulse combination in the entire composition range. Composition analysis of the deposits combined with gravimetric measurements indicated that the $\mathrm{G}$ pulse results in the formation of a deposit with about 90 at.\% Co and 10 at.\% $\mathrm{Pb}$. This was confirmed with electrochemical quartz crystal microbalance experiments by dissolving the two-pulse plated deposits. The $\mathrm{Pb}$ content of the Co-rich deposits when calculated by both methods showed a good agreement.

XRD patterns recorded for two-pulse plated deposits revealed a nanocrystalline structure with grain sizes in the range 5 to $20 \mathrm{~nm}$. The XRD peaks could be well indexed to pure facecentered cubic $\mathrm{Co}$ and $\mathrm{Pb}$. This means that the $\mathrm{Pb}$ codeposited with Co during the $\mathrm{G}$ pulse is not dissolved in Co but is segregated in the form of a nanoscale mixture of the two constituent phases. This observation is in compliance with the immiscibility of $\mathrm{Co}$ and $\mathrm{Pb}$ and the large difference in the lattice distances of the stable crystalline forms of these elements.

Magnetotransport measurements revealed anisotropic magnetoresistance in both d.c. plated and two-pulse plated $\mathrm{Co}-\mathrm{Pb}$ deposits. This means the observed magnetoresistance arises from spindependent electron scattering events dominantly within sufficiently large Co regions (comparable to or larger than the mean free path of electrons in bulk $\mathrm{Co}$ ) and not along electron paths between two $\mathrm{Co}$ regions via the $\mathrm{Pb}$ regions. No indication for a giant magnetoresistance effect due to either a ferromagnetic/non-magnetic layered structure or a granular magnetic structure could be observed.

Low-temperature resistivity measurements revealed a superconducting transition slightly below that of pure $\mathrm{Pb}$. This may be ascribed to a proximity effect: the ferromagnetic Co grains suppress somewhat the superconductivity of the $\mathrm{Pb}$ phase due to the nanoscale phase mixture of the 
two constituents. Some aging effects in the superconducting transition were also observed on a time scale of several months, indicating structural rearrangements in the two-phase mixture of $\mathrm{Co}$ and $\mathrm{Pb}$ that occurs dominantly in the interfaces between the $\mathrm{Co}$ and $\mathrm{Pb}$ regions.

\section{Acknowledgement}

The Hungarian Scientific Research Fund (OTKA) is acknowledged for the support via grant \# K-75008. The authors are indebted to G. Molnar (Natural Science Research Centre, Hung. Acad. Sci.) for preparing the evaporated substrates.

\section{References}

[1] T. B. Massalski (Ed.), Binary Alloy Phase Diagrams, Second Edition Plus Updates on CD-ROM, ASM International, Materials Park, Ohio, USA, 1996.

[2] St. Rashkov, Ts. Dobrev, Z. Noncheva, Y. Stefanov, B. Rashkova, M. Petrova, Lead-cobalt anodes for electrowinning of zinc from sulphate electrolytes, Hydrometallurgy 52 (1999) 223-230.

[3] A. N. Nikoloski, M. J. Nicol, Addition of cobalt to lead anodes used for oxygen evolution - A literature review, Mineral Processing \& Extractive Metall. Rev. 31 (2010) 30-57.

[4] C. Z. Yao, H. X. Ma, X. S. Zhang, L. X. Meng, L. P. Zhao, L. Tai, Y. C. Wang, Q. J. Gong, Y. $\mathrm{X}$. Tong, Electrochemical preparation and magnetic properties of submicron $\mathrm{Co}_{\mathrm{x}} \mathrm{Pb}_{1-\mathrm{x}}$ dendrites, $\mathrm{J}$. Solid State Electrochem. 15 (2011) 1193-1199.

[5] G. B. Ji, S. L. Tang, B. X. Gu, Y. W. Du, Ordered $\mathrm{Co}_{48} \mathrm{~Pb}_{52}$ nanowire arrays electrodeposited in the porous anodic alumina oxide template with enhanced coercivity, J. Phys. Chem. B 108 (2004) 8862-8865.

[6] G. B. Ji, W. Chen, S. L. Tang, B. X. Gu, Z. Li, Y.W. Du, Fabrication and magnetic properties of ordered $20 \mathrm{~mm} \mathrm{Co-Pb}$ nanowire arrays, Solid State Commun. 130 (2004) 541-545.

[7] V. Weihnacht, L. Péter, J. Tóth, J. Pádár, Zs. Kerner, C. M. Schneider, I. Bakonyi, Giant Magnetoresistance in $\mathrm{Co}-\mathrm{CuÕCu}$ Multilayers Prepared by Various Electrodeposition Control Modes, J. Electrochem. Soc. 150 (2003) C507-C515.

[8] L. Péter, J. Pádár, E. Tóth-Kádár, Á. Cziráki, P. Sóki, L. Pogány, I. Bakonyi, Electrodeposition of $\mathrm{Co}-\mathrm{Ni}-\mathrm{Cu} / \mathrm{Cu}$ multilayers 1. Composition, structure and magnetotransport properties, Electrochim. Acta 52 (2007) 3813-3821. 
[9] J. R. Vilche, K. Jüttner, Anion effect on the underpootential depositon of lead on $\mathrm{Cu}(111)$, Electrochim. Acta 32 (1987) 1567-1572.

[10] J. C. Farmer, R. H. Muller, Nucleation of $\mathrm{Pb}$ electrodeposits on $\mathrm{Ag}$ and $\mathrm{Cu}, \mathrm{J}$. Electrochem. Soc. 132 (1985) 39-45.

[11] R. H. Muller, J. C. Farmer, Macroscopic optical model for the ellipsometry of an underpotential deposit: lead on copper and silver, Surf. Sci. 135 (1983) 521-531.

[12] H. Siegenthaler, K. Jüttner, Voltammetric investigation of lead adsorption on Cu(111) singlecrystal substrates, J. Electroanal. Chem. 163 (1984) 327-343.

[13] I. Bakonyi, L. Péter, Electrodeposited multilayer films with giant magnetoresistance (GMR): Progress and problems, Progr. Mater. Sci. 55 (2010) 107-245.

[14] L. Péter, Q. X. Liu, Z. Kerner, I. Bakonyi, Relevance of the potentiodynamic method in parameter selection for pulse-plating of $\mathrm{Co}-\mathrm{Cu} / \mathrm{Cu}$ multilayers, Electrochimica Acta 49 (2004) 1513-1526.

[15] T. Spassov, L. Lyubenova, Y. Liu, S. Bliznakov, M. Spassova, N. Dimitrov, Mechanochemical synthesis, thermal stability and selective electrochemicaldissolution of $\mathrm{Cu}-\mathrm{Ag}$ solid solutions, J. Alloys and Compd. 478 (2009) 232-236.

[16] I. McCue, J. Snyder, X. Li, Q. Chen, K. Sieradzki, J. Erlebacher, Apparent Inverse GibbsThomson Effect in Dealloyed Nanoporous Nanoparticles, Phys. Rev. Lett. 108 (2012) Art. No. 225503(1-5).

[17] K. Sieradzki, N. Dimitrov, D. Movrin, C. McCall, N. Vasiljevic, J. Erlebacher, The Dealloying Critical Potential, J. Electrochem. Soc. 149 (2002) B370-B377.

[18] B. D. Cullity, S. R. Stock, Elements of X-Ray Diffraction, third ed., Prentice-Hall Inc., New Jersey, 2001.

[19] C. Michaelsen, On the structure and homegeneity of solid solutions - The limts of conventional X-ray diffraction, Philos. Mag. A 72 (1995) 813-828.

[20] T. R. McGuire, R. I. Potter, Anisotropic magnetoresistance in ferromagnetic 3d alloys, IEEE Trans. Magn. 11 (1975) 1018-1038.

[21] I. Bakonyi, E. Tóth-Kádár, J. Tóth, L. F. Kiss, L. Pogány, Á. Cziráki, C. Ulhaq-Bouillet, V. Pierron-Bohnes, A. Dinia, B. Arnold, K. Wetzig, Room temperature electronic transport properties of Co metal and $\mathrm{Co}(\mathrm{Ru})$ dilute alloys Europhys. Lett. 58 (2002) 408-414.

[22] T. R. McGuire, W. D. Grobman, D. E. Eastman, Photoemission Studies of Ni-Co Alloys Having Large Anisotropic Magnetoresistance, AIP Conf. Proc. 18 (1974) 903-907.

[23] C. Kittel, Introduction to Solid State Physics, Wiley, New York, 1986. 
[24] I. Bakonyi, E. Simon, B. G. Tóth, L. Péter, L. F. Kiss, Giant magnetoresistance in electrodeposited $\mathrm{Co}-\mathrm{Cu} / \mathrm{Cu}$ multilayers: Origin of the absence of oscillatory behavior, Phys. Rev. B 79 (2009) 174421/1-13.

[25] P. Koorevaar, Y. Suzuki, R. Coehoorn, J. Aarts, Decoupling of superconducting V by ultrathin Fe layers in V/Fe multilayers, Phys. Rev. B 49 (1994) 441-449.

[26] J. Aarts, J. M. E. Geers, E. Brück, A. A. Golubov, R. Coehoorn, Interface transparency of superconductor/ferromagnetic multilayers Phys. Rev. B 56 (1997) 2779-2787.

[27] B. P. Vodopyanov, L. R. Tagirov, H. Z. Durusoy, A. V. Berezhnov, The influence of alloying or interdiffusion on the superconducting properties of ferromagnet/superconductor layered systems Physica C 366 (2001) 31-42. 
Table 1

Composition of the electrolytes used for Pb-Co codeposition experiments.

\begin{tabular}{|l|c|c|c|}
\hline & \multicolumn{3}{|c|}{ Concentration / mol dm } \\
Source compound & Acetate bath & Nitrate bath & Chloride bath \\
\hline $\mathrm{Co}\left(\mathrm{CH}_{3} \mathrm{COO}\right)_{2} \bullet 4 \mathrm{H}_{2} \mathrm{O}$ & 0.4 & --- & --- \\
\hline $\mathrm{Pb}\left(\mathrm{CH}_{3} \mathrm{COO}\right)_{2} \cdot 3 \mathrm{H}_{2} \mathrm{O}$ & 0.013 & --- & 0.006 \\
\hline $\mathrm{CH}_{3} \mathrm{COOH}$ & 0.1 & --- & --- \\
\hline $\mathrm{Co}\left(\mathrm{NO}_{3}\right)_{2} \bullet 6 \mathrm{H}_{2} \mathrm{O}$ & --- & 0.4 & --- \\
\hline $\mathrm{Pb}\left(\mathrm{NO}_{3}\right)_{2} \bullet 3 \mathrm{H}_{2} \mathrm{O}$ & --- & 0.013 & --- \\
\hline $\mathrm{CoCl} \mathrm{Cl}_{2} \cdot 6 \mathrm{H}_{2} \mathrm{O}$ & --- & --- & 0.4 \\
\hline $\mathrm{KCl}$ & --- & --- & 2.5 \\
\hline $\mathrm{pH}$ of the bath & 5.15 & 4.55 & 4.55 \\
\hline
\end{tabular}

\section{Table 2}

List of the two-pulse plated samples used in the low-temperature resistivity measurements.

\begin{tabular}{|l|c|c|c|c|}
\hline \multirow{2}{*}{} & \multicolumn{5}{|c|}{ Sample code } \\
\cline { 2 - 5 } & $\mathrm{A}$ & $\mathrm{B}$ & $\mathrm{C}$ & $\mathrm{D}$ \\
\hline$t(\mathrm{Co}) / \mathrm{s}$ & 0.27 & 0.195 & 0.135 & 0.075 \\
\hline Equivalent Co layer thickness / nm & 3.0 & 2.15 & 1.5 & 0.85 \\
\hline$j(\mathrm{Co}) / \mathrm{mAcm}^{-2}$ & \multicolumn{5}{|c|}{-32} \\
\hline$E(\mathrm{~Pb}) / \mathrm{V}$ vs. SCE & \multicolumn{5}{|c|}{-9.62} \\
\hline$Q(\mathrm{~Pb}) / \mathrm{mCcm}^{-2}$ & \multicolumn{5}{|c|}{6.0} \\
\hline Equivalent $\mathrm{Pb}$ layer thickness / $\mathrm{nm}$ & 100 & 110 & 120 & 131 \\
\hline Number of repeat periods & \multicolumn{5}{|c|}{ Acetate bath (see Table 1) } \\
\hline Total sample thickness / $\mathrm{nm}$ & \multicolumn{5}{|c|}{} \\
\hline Electrolyte used & \multicolumn{5}{|c|}{} \\
\hline
\end{tabular}




\section{Figures}

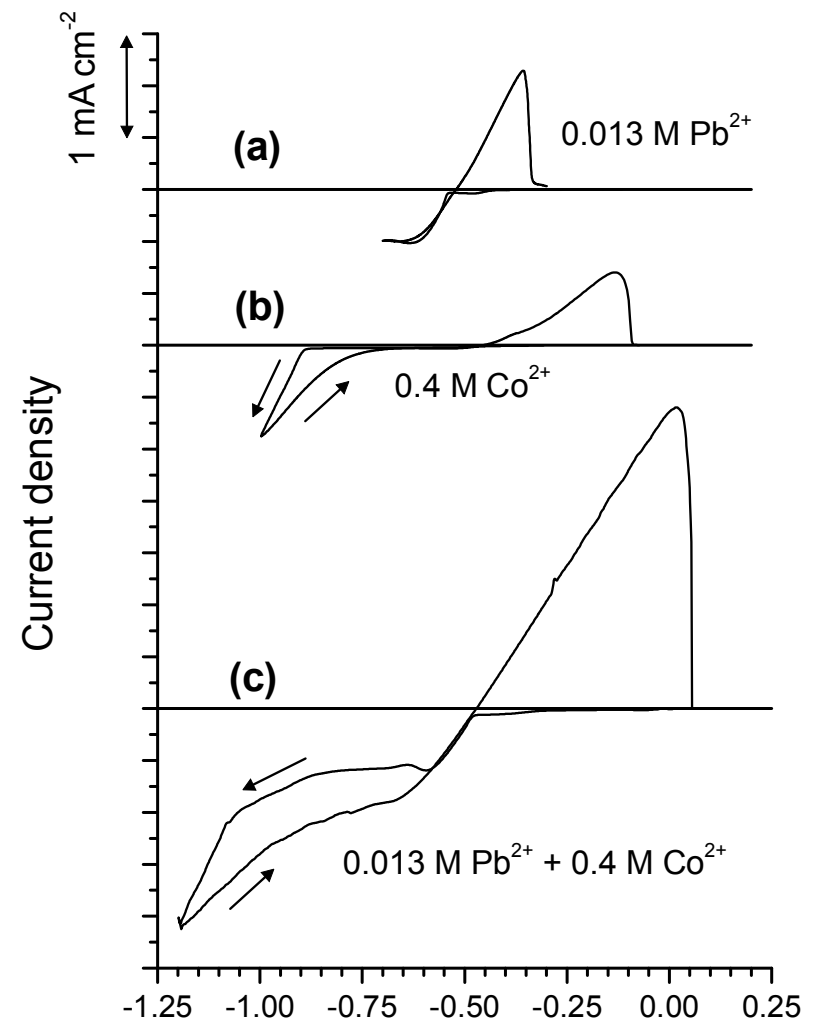

Figure 1 - Cyclic voltammograms recorded for the acetate bath in the presence of $\mathrm{Pb}^{2+}$ (a), $\mathrm{Co}^{2+}$ (b) and both (c). Scan rate, $2 \mathrm{mV} / \mathrm{s}$. Arrows indicate the scan direction.

\section{$E / V$ vs. SCE}

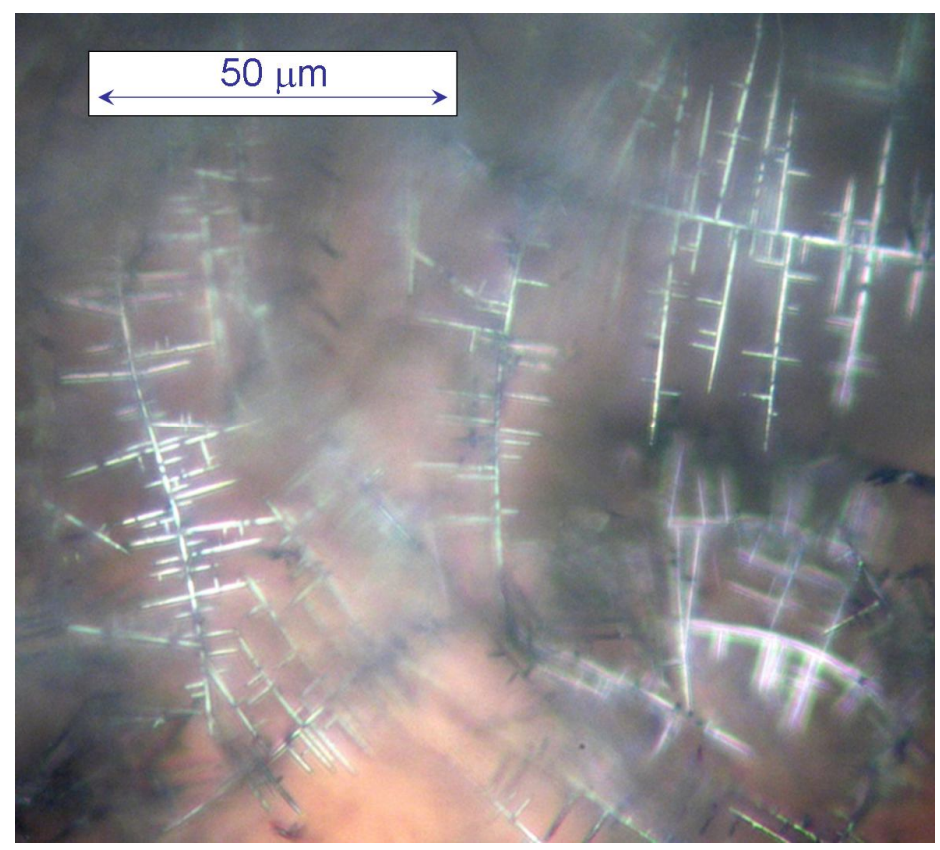

Figure 2 - Optical microscopy picture of the $\mathrm{Pb}$ dendrites grown at $-4.5 \mathrm{mAcm}^{-2}$ current density from the acetate bath. The picture was exposed while the dendritic deposit was under a drop of water. 
Figure 3

SEM pictures obtained for samples produced with potentiostatic condition from the acetate bath. Deposition potential: $E=-0.65 \mathrm{~V}$ (a) and $E=-1.025 \mathrm{~V}(\mathrm{~b})$.
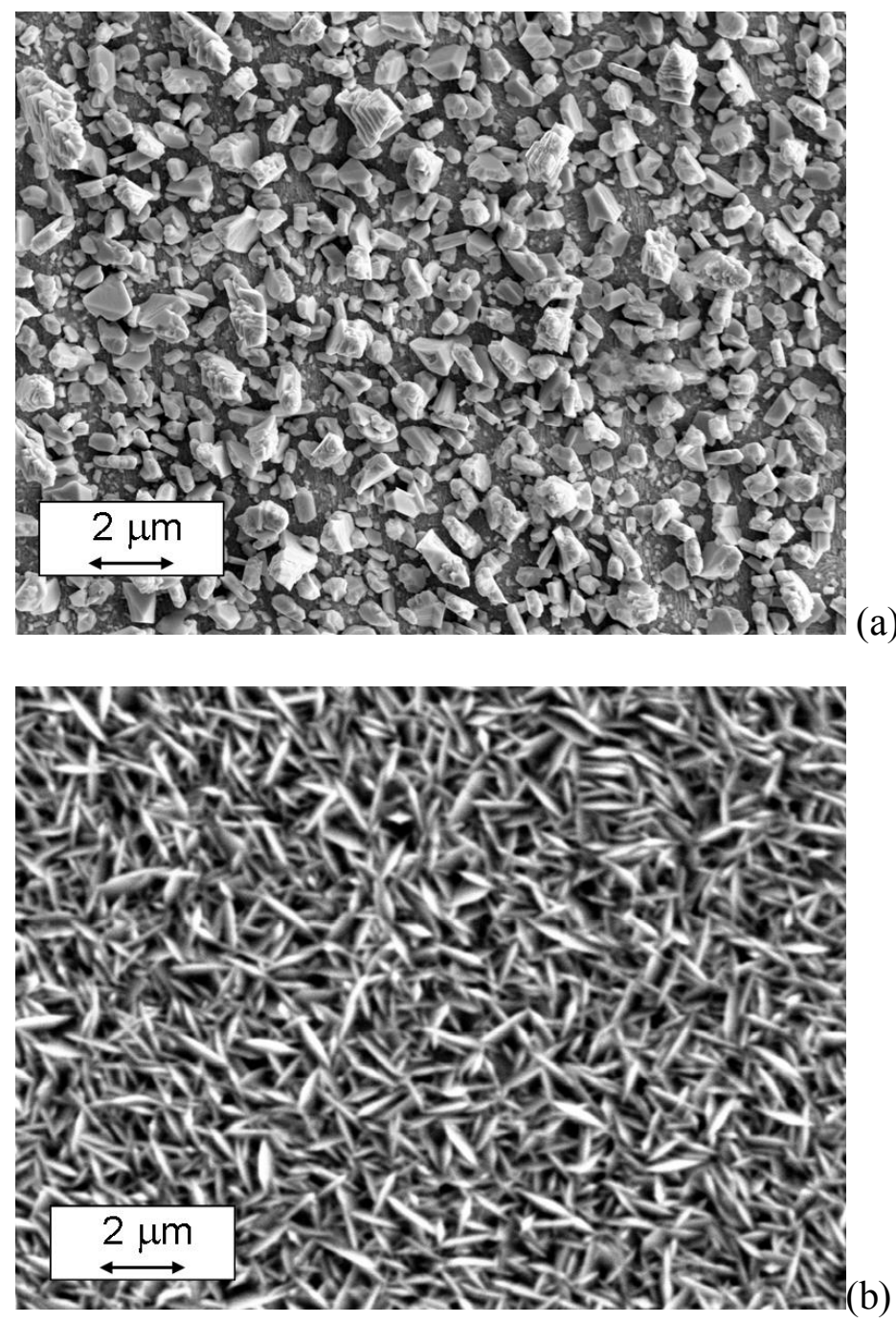
Figure 4

(a) Composition of the two-pulse plated $\mathrm{Co}-\mathrm{Pb}$ deposits from the acetate bath as a function of the $\mathrm{Pb}$ deposition potential $\left(E_{\mathrm{Pb}}\right)$. Deposition conditions: $j=-32 \mathrm{mAcm}^{-2}$ and $t=0.28 \mathrm{~s}$ (G pulse, equivalent Co layer thickness: $3 \mathrm{~nm}$ ); $Q=-3.2 \mathrm{mCcm}^{-2}$ (P pulse, equivalent $\mathrm{Pb}$ layer thickness: 3 $\mathrm{nm}$ ); total deposit thickness: $900 \mathrm{~nm}$. Dashed lines serve as a guide for the eye only.

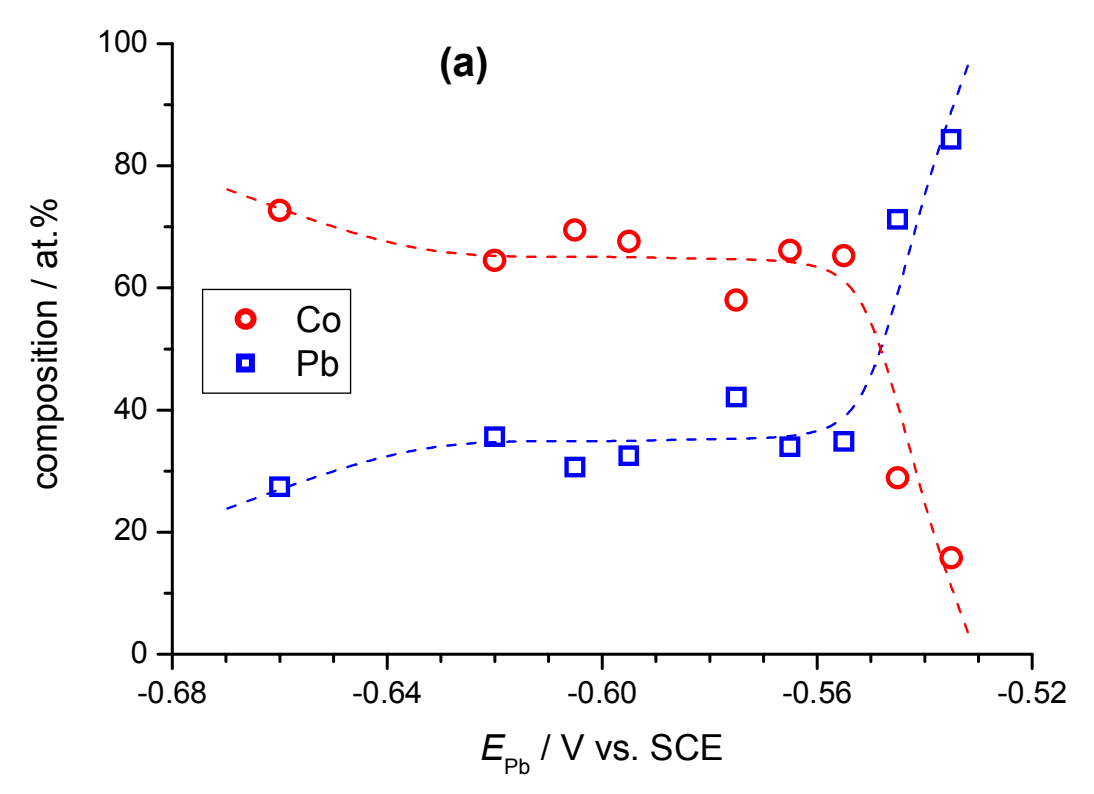

(b) Current transients recorded during the P pulse with different $E_{\mathrm{Pb}}$ values (see next the curves) as a function of the time passed after the pulse switch. The samples are the same as those used for the composition analysis. The inset shows the polarization curve recorded at $2 \mathrm{mV} / \mathrm{s}$ (solid line) and the current obtained at the end of the P pulse during the two-pulse plating experiments.

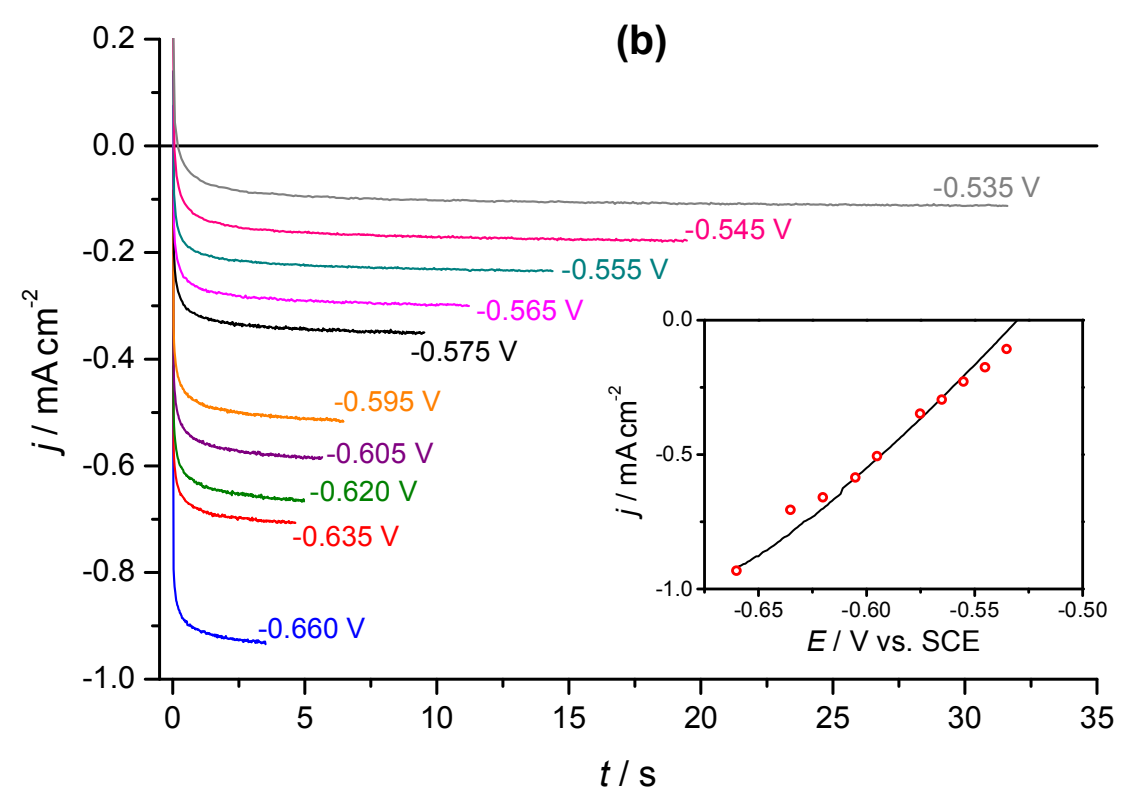


Figure 5

SEM pictures obtained for pulse-plated samples from the acetate bath. Deposition parameters are the same as for samples shown in Fig. 6. The value of the deposition potential in the P pulse is indicated below each image.

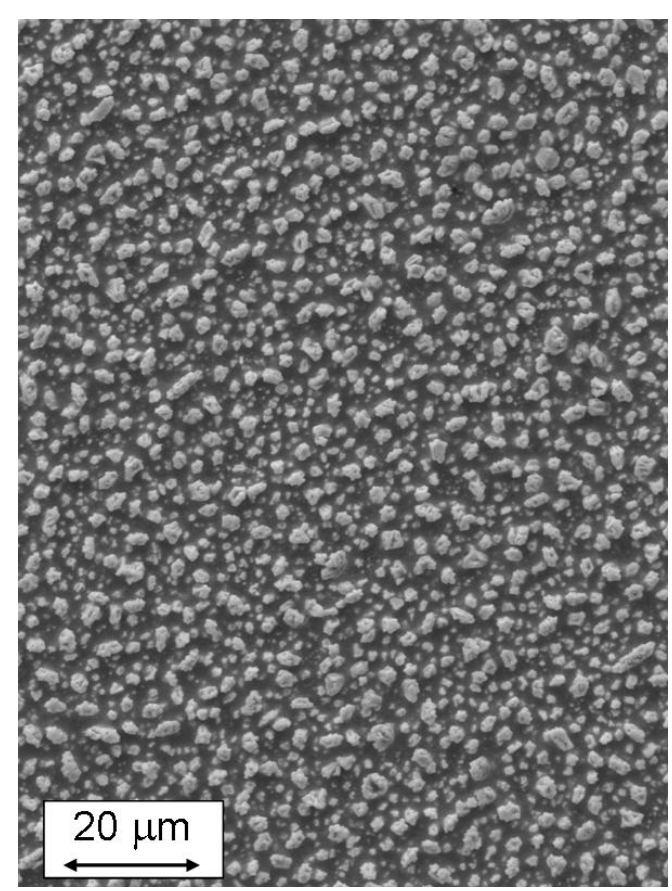

$-0.62 \mathrm{~V}$

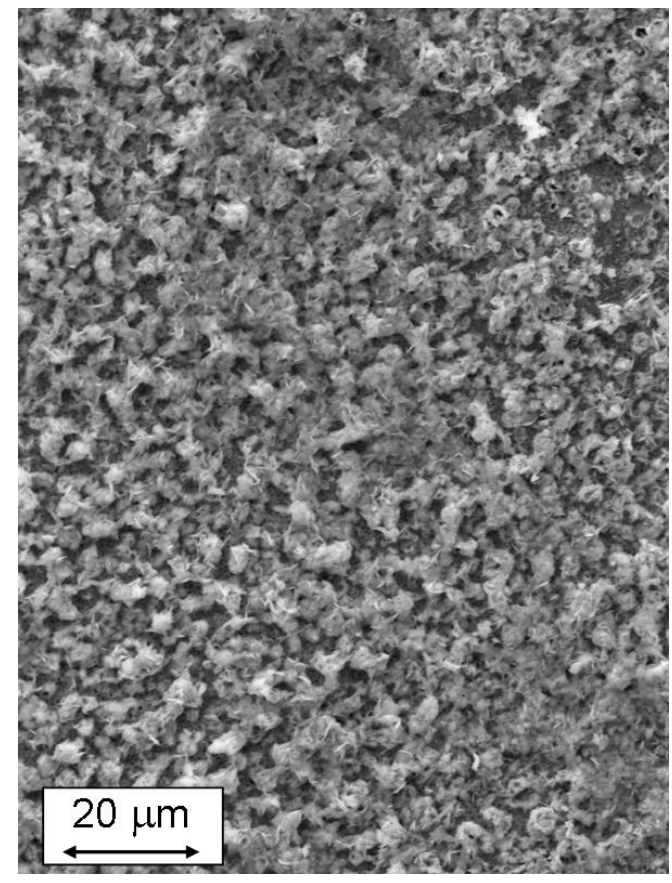

$-0.555 \mathrm{~V}$

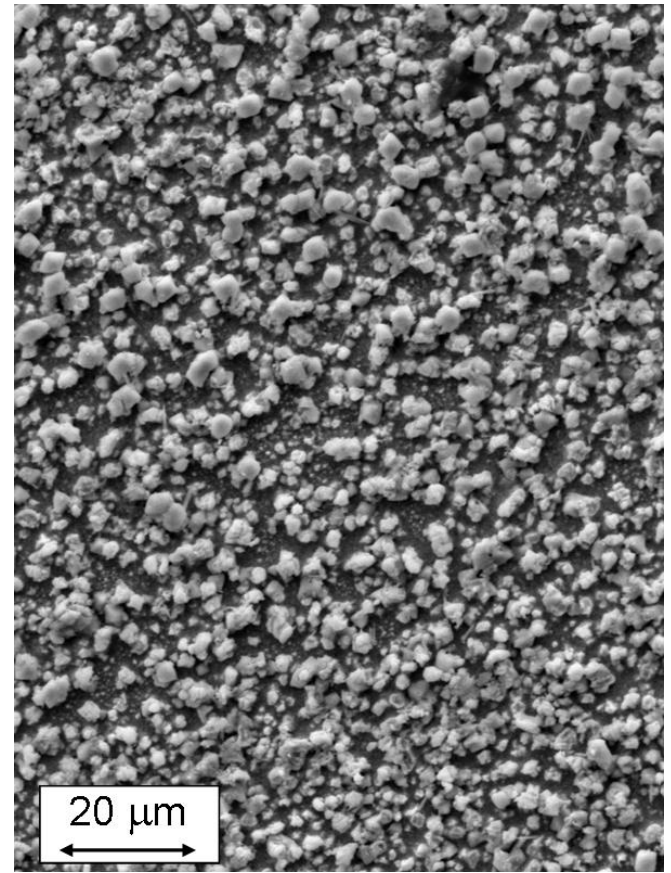

$-0.575 \mathrm{~V}$

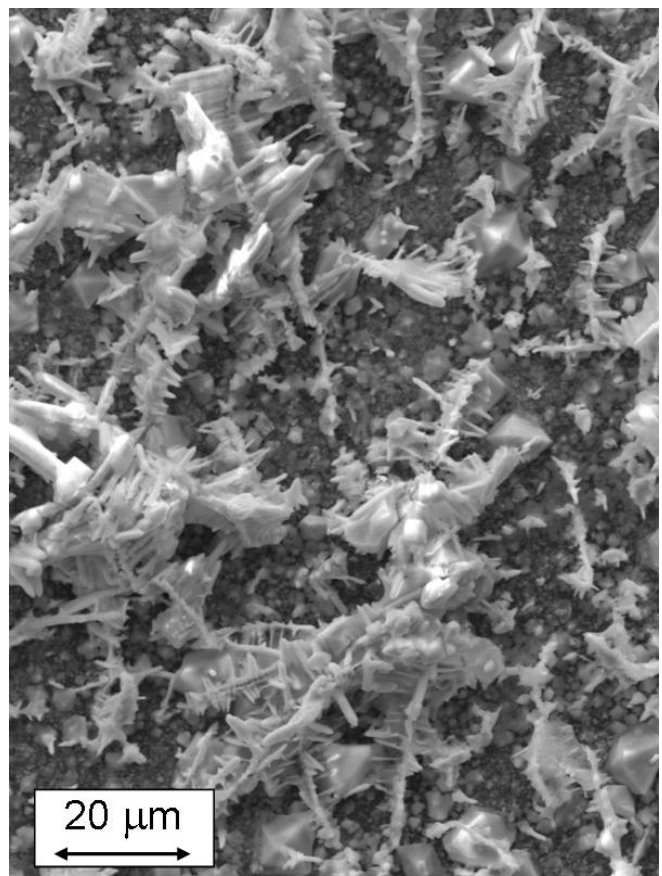

$-0.535 \mathrm{~V}$ 
Figure 6

Composition of the two-pulse plated $\mathrm{Co}-\mathrm{Pb}$ deposits from the acetate bath as a function of the $\mathrm{Co}$ deposition current density. Deposition conditions: $j_{\mathrm{Co}} t_{\mathrm{Co}}=-8.95 \mathrm{mCcm}^{-2}$, (G pulse, equivalent $\mathrm{Co}$ layer thickness: $3 \mathrm{~nm}$ ); $E_{\mathrm{Pb}}=-0.62 \mathrm{~V}, Q_{\mathrm{Pb}}=-3.2 \mathrm{mCcm}^{-2}$ (P pulse, equivalent $\mathrm{Pb}$ layer thickness: 3 $\mathrm{nm}$ ); deposit thickness: $900 \mathrm{~nm}$. Dashed lines serve as a guide for the eye only.

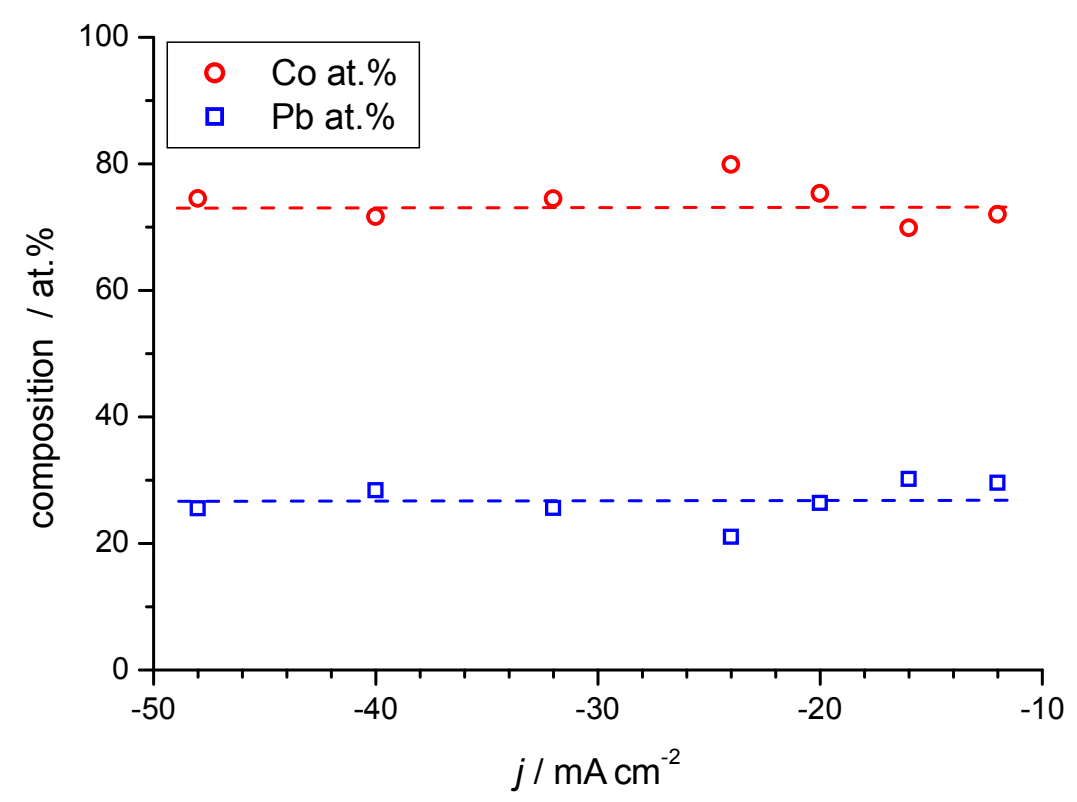


Figure 7

Composition (a) and weight (b) of the two-pulse plated $\mathrm{Co}-\mathrm{Pb}$ deposits from the acetate bath as a function of the Co deposition current density. Deposition conditions: $j_{\mathrm{Co}}=-32 \mathrm{mAcm}^{-2}, t_{\mathrm{Co}}=0.28 \mathrm{~s}$ (G pulse, equivalent Co layer thickness: $3 \mathrm{~nm}$ ); $E_{\mathrm{Pb}}=-0.62 \mathrm{~V}$, deposit thickness: $900 \mathrm{~nm}$ and 1800 $\mathrm{nm}$ for composition analysis and gravimetric measurements, respectively. Large open symbols refer to the experimental data, while solid symbols connected with a dashed line show the result of the calculations with the following parameters: $\eta_{\mathrm{P}}=1, \eta_{\mathrm{G}}=0.97$ and $y_{\mathrm{Pb}}^{*}=0.1$.
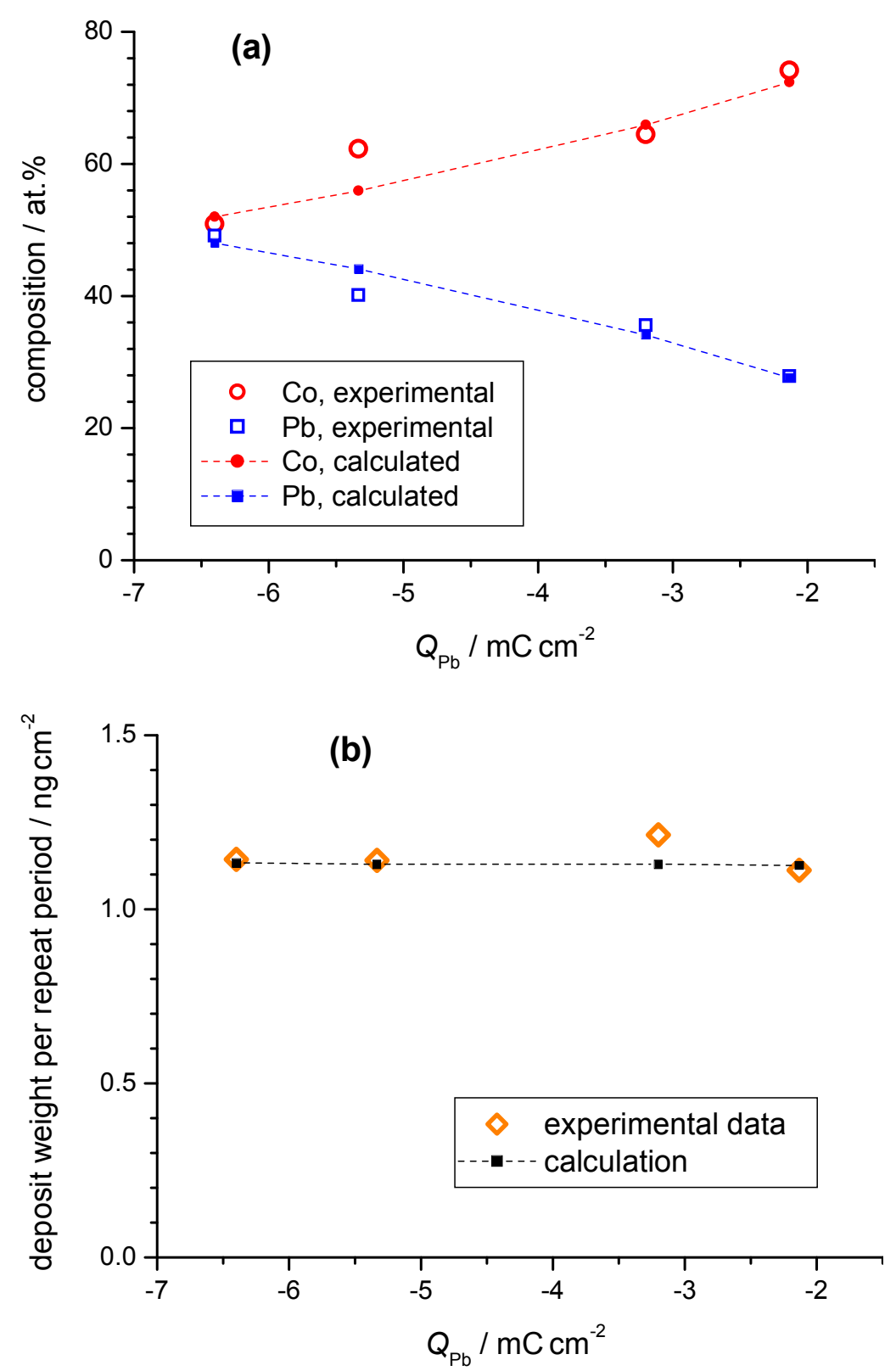


\section{Figure 8}

Voltammogram recorded for the dissolution of a sample produced with two-pulse plating (bottom curve) and the derivative of the quartz crystal microbalance recorded in parallel to the voltammogram (top curve). Scan rate during the dissolution: $0.8 \mathrm{mVs}^{-1}$. Deposition conditions: $E_{\mathrm{Pb}}$ $=-0.62 \mathrm{~V}, Q_{\mathrm{Pb}}=-8 \mathrm{mC} ; I_{\mathrm{Co}}=-32 \mathrm{~mA}, t=0.27 \mathrm{~s}$ (nominal layer thicknesses are $3 \mathrm{~nm}(\mathrm{Co})$ and $5 \mathrm{~nm}$ $(\mathrm{Pb})) ; N=40$.

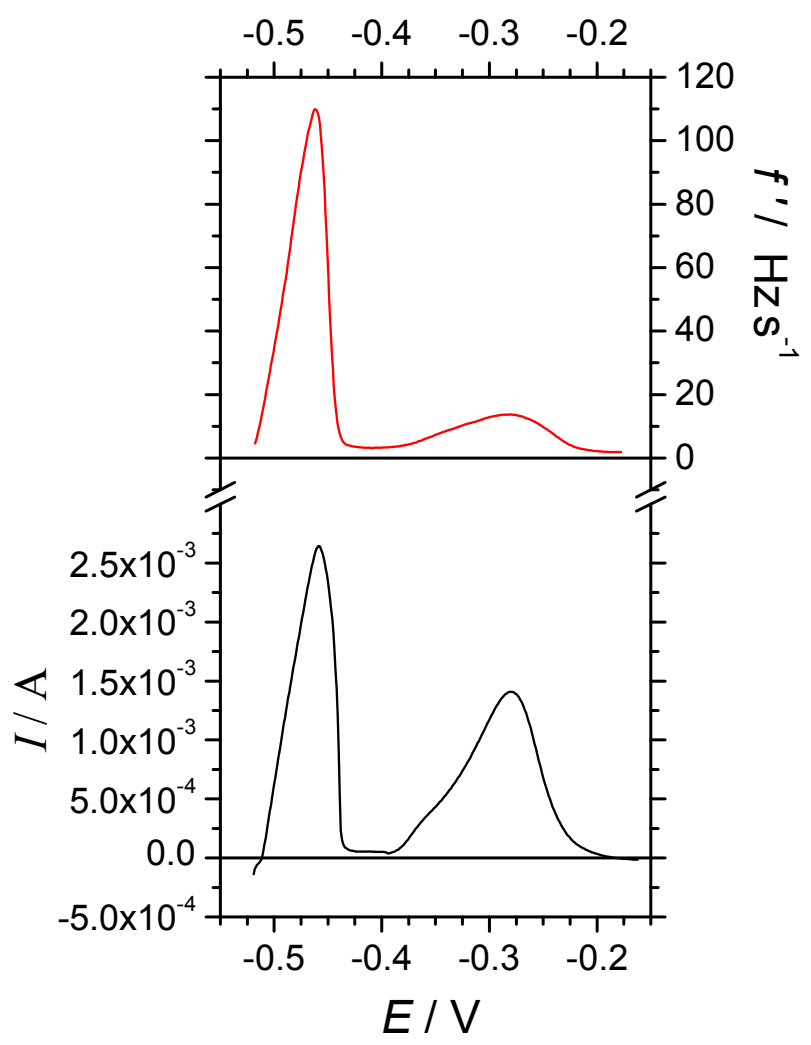




\section{Figure 9}

Typical X-ray diffractogram of two-pulse plated deposits prepared from the acetate bath. The present G/P sample was prepared under the following conditions: in the G pulse, $j=-32 \mathrm{mAcm}^{-2}$ and $t=0.27 \mathrm{~s}$ (equivalent Co layer thickness: $3 \mathrm{~nm}$ ); in the P pulse: $Q=-1.067 \mathrm{mCcm}^{-2}, E_{\mathrm{Pb}}=-620$ $\mathrm{mV}$ (equivalent $\mathrm{Pb}$ layer thickness: $1 \mathrm{~nm}$ ); bilayer number: 225; the total deposit thickness was 900 nm.

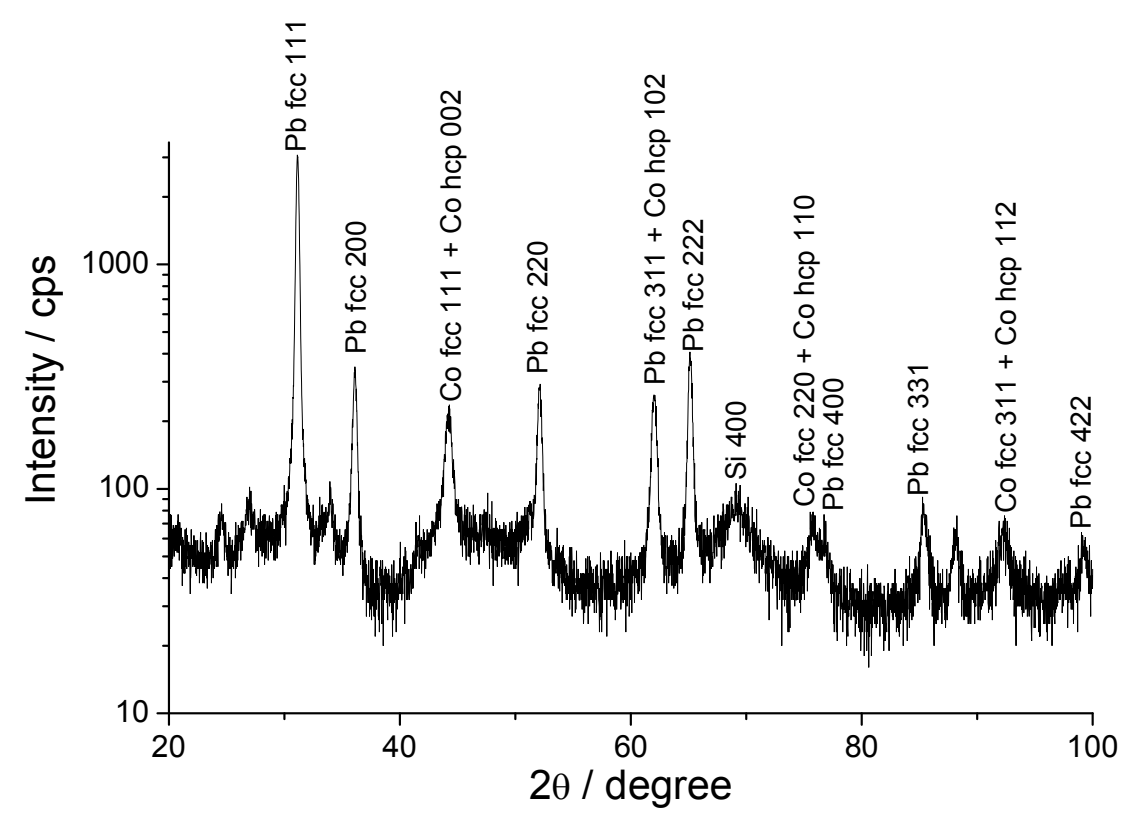


Figure 10

Room-temperature magnetoresistance curves obtained for samples prepared under various deposition conditions from the acetate bath. (a) d.c. plated Co sample prepared from an acetate bath void of $\mathrm{Pb}^{2+}$; (b) d.c. plated $\mathrm{Co}-\mathrm{Pb}$ sample prepared from an acetate bath containing also $\mathrm{Pb}^{2+}$; for both samples prepared with d.c. plating: $j=-32 \mathrm{mAcm}^{-2}$. (c) two-pulse plated $\mathrm{Co}-\mathrm{Pb}$ sample prepared in the G/P mode: $j=-32 \mathrm{mAcm}^{-2}$ and $t=0.28 \mathrm{~s}$ (G pulse, equivalent Co layer thickness: 3 $\mathrm{nm}) ; Q=-3.2 \mathrm{mCcm}^{-2}, E_{\mathrm{Pb}}=-620 \mathrm{mV}$ (P pulse, equivalent $\mathrm{Pb}$ layer thickness: $3 \mathrm{~nm}$ ). Solid symbols: longitudinal MR, open symbols: transverse MR.

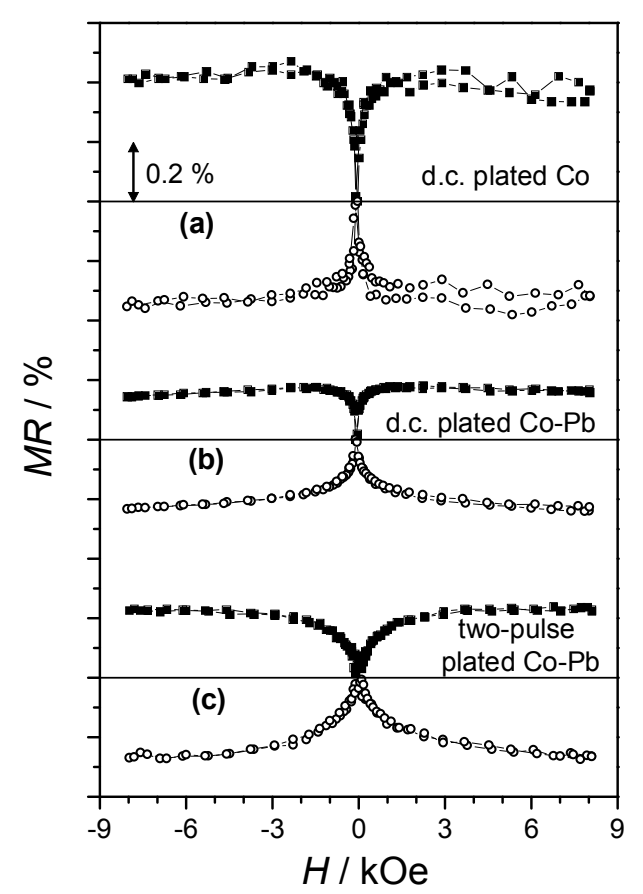


Figure 11

Low-temperature normalized resistance of the aged samples on a log-log scale. The inset shows the normalized resistivity on a lin-lin scale for sample $\mathrm{C}$ for which the superconducting transition is also clearly visible (the negative normalized resistivity values appear due to the choice of $R_{\mathrm{O}}$ corresponding to the residual resistivity of the normal state).

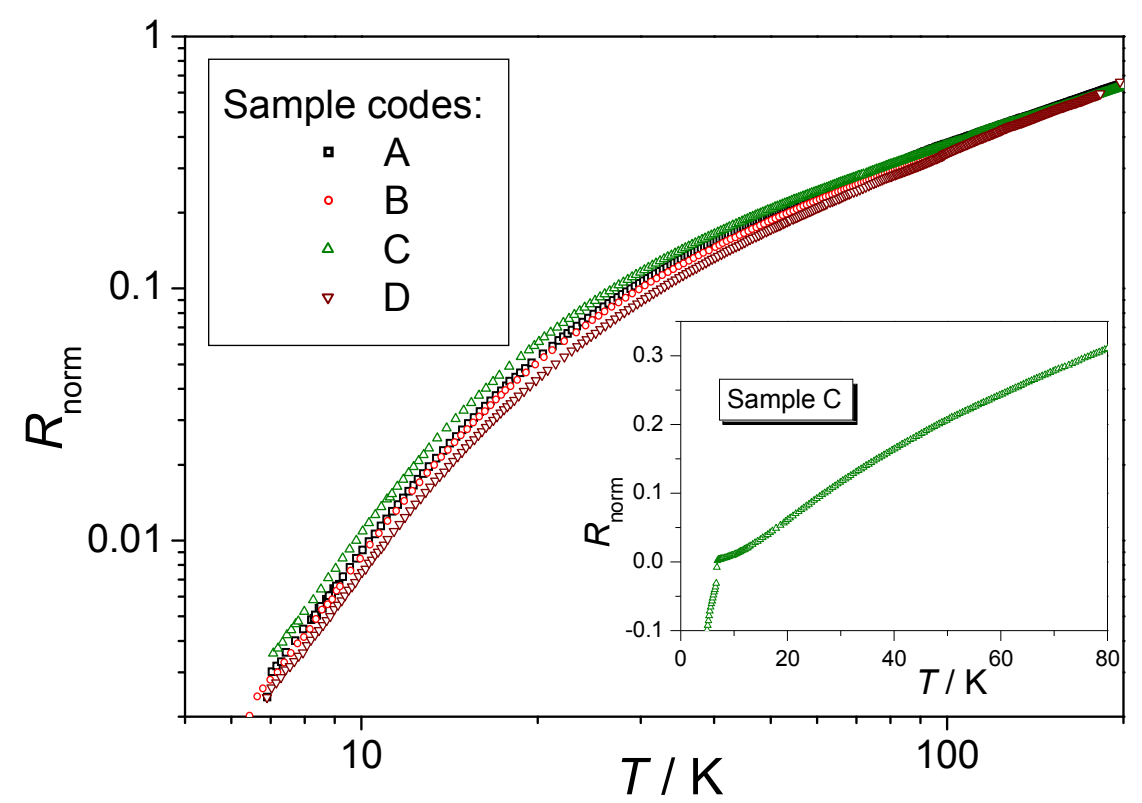

Figure 12

Low-temperature normalized resistance of sample B for fresh and aged conditions. Note the fully superconducting state for the fresh sample below $5.8 \mathrm{~K}$ in spite of the presence of a significant amount of ferromagnetic Co grains in the sample.

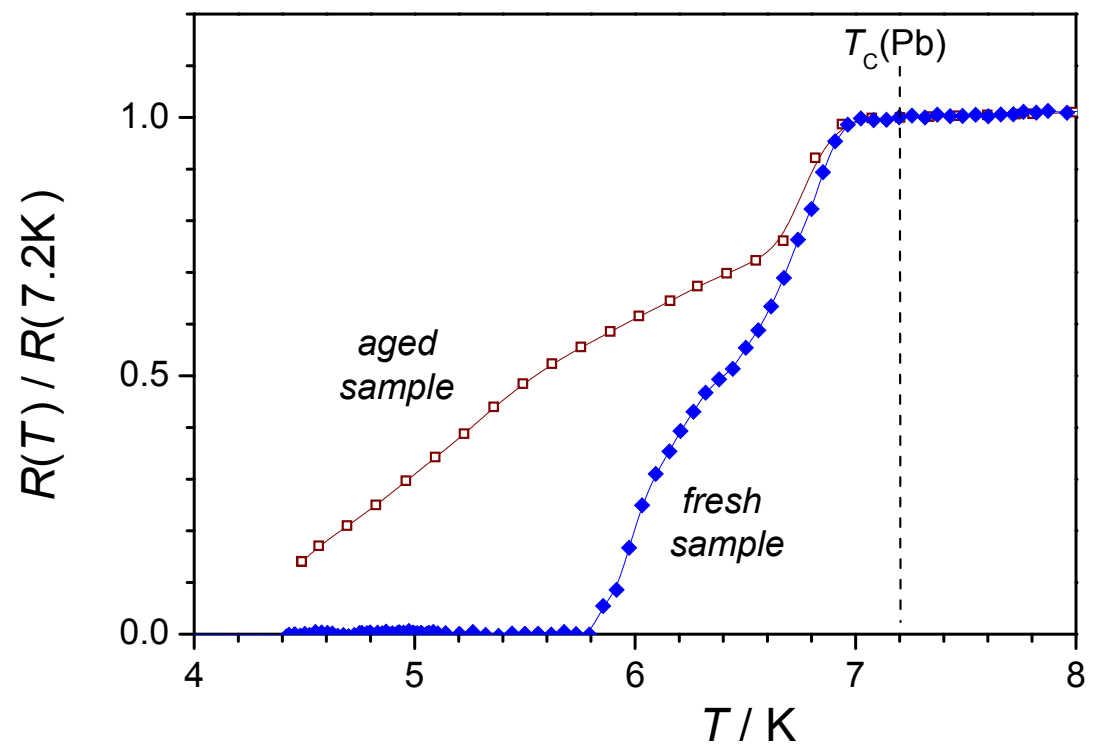


Figure 13

Low-temperature normalized resistance of sample A for the fresh state. The inset shows the difference between the fresh and aged states.

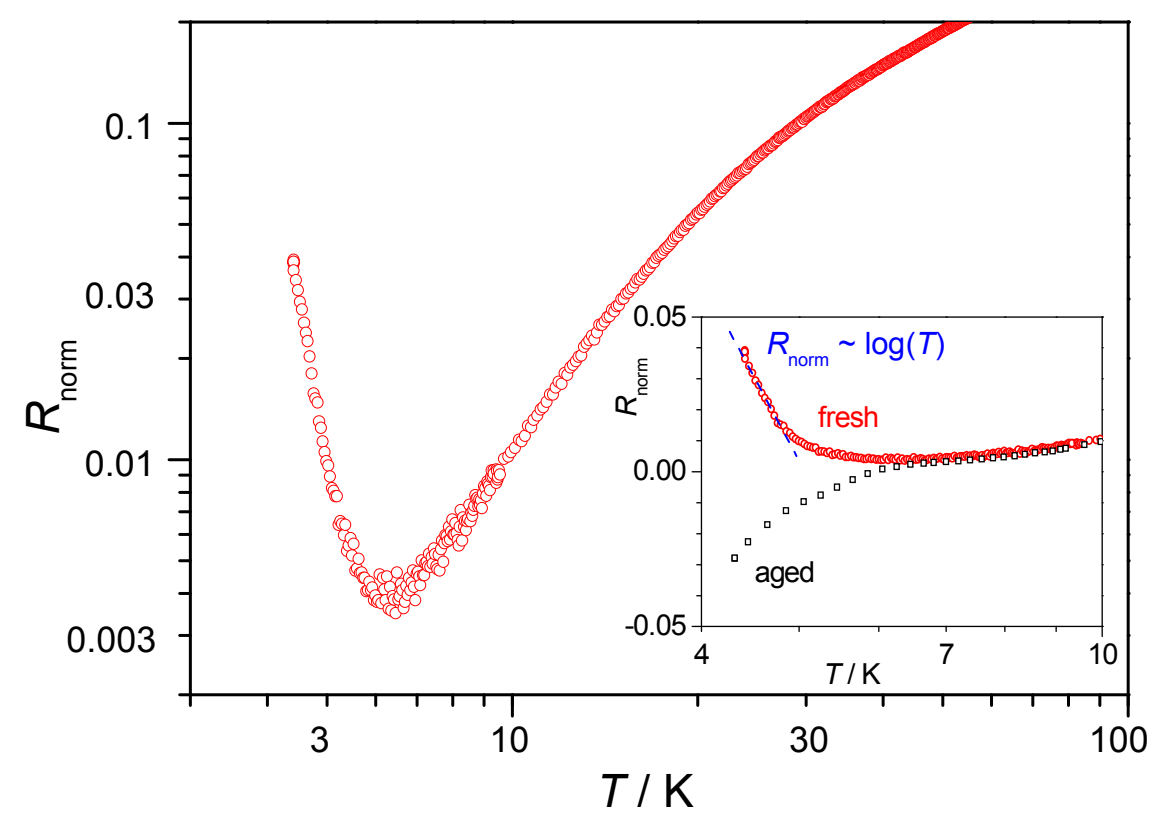

